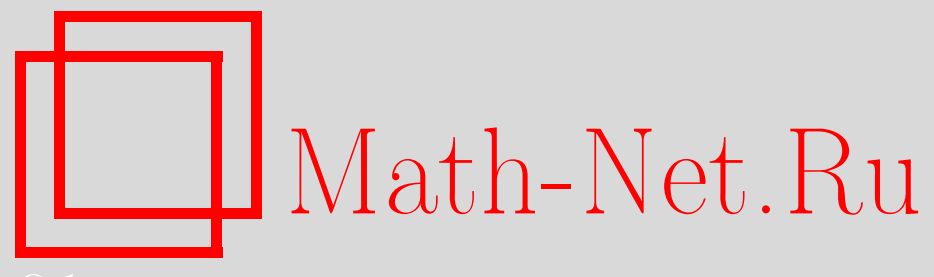

A. Yu. Zaitsev, Multidimensional Version of a Result of Sakhanenko in the Invariance Principle for Vectors with Finite Exponential Moments. III, Теория вероятн. и ее примен., 2001, том 46, выпуск 4, 744-769

DOI: https://doi.org/10.4213/tvp3798

Использование Общероссийского математического портала MathNet.Ru подразумевает, что вы прочитали и согласны с пользовательским соглашением

http://www . mathnet.ru/rus/agreement

Параметры загрузки:

IP : 3.91 .87 .62

26 апреля 2023 г., 18:13:23

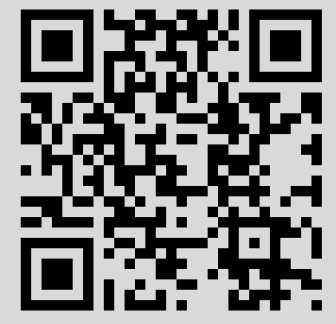




\section{MULTIDIMENSIONAL VERSION OF A RESULT OF SAKHANENKO IN THE INVARIANCE PRINCIPLE FOR VECTORS WITH FINITE EXPONENTIAL MOMENTS. III ${ }^{1)}$}

Получен многомерный вариант результата Саханенко [18] о гауссовской аппроксимации последовательности сумм независимых случайных векторов с конечными экспоненциальными моментами.

Ключевые слова и фразы: многомерный принцип инвариантности, сильная аппроксимация, суммы независимых разнораспределенных случайных векторов.

9. Estimates in the dyadic scheme in the case of coinciding moments of the first three orders.

Lemma 9.1. Let $\tau \geqslant 1$ and let Conditions $\mathbf{A}(\tau), \mathbf{B}, \mathbf{D}_{\Psi}$ and $\mathbf{C}_{\Psi}(p)$ be satisfied with some $p, 1 \leqslant p \leqslant N$, for the dyadic scheme described in Sections 5-7. Then there exist absolute positive constants $c_{57}, c_{58}$ such that

a) if $\tau^{2} d^{3} p / 2^{N-p} \leqslant c_{57}$, then

$$
\left\|U_{N, 1}-V_{N, 1}\right\| \leqslant c_{58} \tau^{2} \cdot 2^{-N / 2}\left(d^{5 / 2}+d \cdot 2^{-3 N / 2}\left\|U_{N, 1}\right\|^{3}\right)
$$

provided that $\left\|U_{N, 1}\right\| \leqslant c_{57 \cdot 2^{N}} /\left(d^{3 / 2} \tau\right)$;

b) if $1 \leqslant n \leqslant N, 1 \leqslant k \leqslant 2^{N-n}, \tau^{2} d^{3} p / 2^{n-p} \leqslant c_{57}$, then

$$
\begin{aligned}
\left\|\widetilde{\mathscr{U}}_{n, k}-\widetilde{\mathscr{V}}_{n, k}\right\| \leqslant & \frac{c_{58} \tau^{2}}{2^{n / 2}}\left(d^{5 / 2}+d \cdot 2^{-3 n / 2}\left\|\mathbf{U}_{n, k}\right\|^{3}\right) \\
& +\frac{1}{8 c_{17}}\left\|U_{n, k}-V_{n, k}\right\|
\end{aligned}
$$

provided that $\max \left\{\left\|\mathbf{U}_{n, k}\right\|,\left\|V_{n, k}\right\|\right\} \leqslant c_{57} 2^{n} /\left(d^{3 / 2} \tau\right)$.

${ }^{*}$ С.-Петербургское отделение Математического института им. В. А. Стеклова PАН, ул. Фонтанка, 27, 191011 С.-Петербург, Россия; e-mail: zaitsev@pdmi.ras.ru

1) Research was supported by the SFB 343 in Bielefeld, by the Russian Foundation of Basic Research, grant 96-01-00672, by grant INTAS-RFBR 95-0099 and by grant RFBRDFG 96-01-00096-ge. Ч. I, II - см. Теория вероятн. и ее примен., 2000, т. 45, в. 4, c. $718-738 ; 2001$, т. 46 , в. 3 , с. $535-561$. 
P r o of. From Lemma 7.2, from the definition of the distributions $\Psi(\cdot)$ and from Conditions $\mathbf{B}_{1}, \mathbf{D}_{\Psi}$ and $\mathbf{C}_{\Psi}(p)$ it follows that the vectors $\xi_{k}, X_{k}$ and $Y_{k}$ have the same moments of the first three orders for each $k=1, \ldots, 2^{N}$. Using the linearity and independence arguments, we see that the same can be said about corresponding moments of the random vectors $\mathbf{U}=\left\{U_{n, k}, \widetilde{\mathbf{U}}_{n, k}, \mathbf{U}_{n, k}, \mathscr{U}_{n, k}, \mathscr{U}\right\}$ and $\mathbf{V}=\left\{V_{n, k}, \tilde{\mathbf{V}}_{n, k}, \mathbf{V}_{n, k}, \mathscr{V}_{n, k}, \mathscr{V}\right\}$ from Remark 6.3.

Let us prove at first the statement a). By condition B (see (6.13) and (6.14)), the coordinates $\mathscr{U}^{(1)}, \ldots, \mathscr{U}^{(d)}$ of the vectors $\mathscr{U}=\mathbb{J}_{N, 1} U_{N, 1}$ and $\mathscr{U}^{j}=\overline{\mathbb{P}}_{j}(\mathscr{U}), j=1, \ldots, d$, are noncorrelated and have mean zero and the same variance $2^{N}$. The same is valid for the coordinates $\mathscr{V}^{(1)}, \ldots, \mathscr{V}^{(d)}$ of the vectors $\mathscr{V}=\mathbb{J}_{N, 1} V_{N, 1}$ and $\mathscr{V}^{j}=\overline{\mathbb{P}}_{j}(\mathscr{V})$. By Corollary 7.1,

$$
\mathscr{L}\left(\mathscr{U}^{j}\right), \mathscr{L}\left(\mathscr{V}^{j}\right) \in \mathscr{A}_{j}^{*}\left(c_{30} \tau, 4,4\right), \quad j=1, \ldots, d,
$$

if $\tau \geqslant 1$ and $\tau^{2} d^{3}(p+1) / 2^{N-p} \leqslant c_{29}$. Thus, the distributions $\mathscr{L}\left(\mathscr{U}^{j}\right), \mathscr{L}\left(\mathscr{V}^{j}\right)$ satisfy in the $j$-dimensional case the conditions of Lemma 3.3 with $\operatorname{cov} \xi_{k}=$ $\operatorname{cov} \mathscr{U}^{j}=2^{N} \mathbb{I}_{j}, \mathbb{B}=\operatorname{cov} \mathscr{U}^{j-1}=2^{N} \mathbb{I}_{j-1}$ (the last equality for $j \geqslant 2$ ) and with the change of $\tau$ by $c_{30} \tau$ provided that $c_{57} \leqslant c_{29} / 2$.

Taking into account (5.14), (5.15) and applying Lemmas 3.3, 8.2 in the $j$-dimensional case, $j=1, \ldots, d$, we obtain that

$$
\left|\mathscr{U}^{(1)}-\mathscr{V}^{(1)}\right| \leqslant \frac{c \tau^{2}}{2^{N / 2}}\left(1+\frac{\left|\mathscr{U}^{(1)}\right|^{3}}{2^{3 N / 2}}\right),
$$

provided that $\tau / 2^{N / 2} \leqslant c_{59},\left|\mathscr{U}^{(1)}\right| \leqslant c_{59} \cdot 2^{N} / \tau$. Moreover, for $2 \leqslant j \leqslant d$,

$$
\begin{aligned}
\left|\mathscr{U}^{(j)}-\mathscr{V}^{(j)}\right| \leqslant & \frac{c \tau^{2}}{2^{N / 2}}\left(j^{2}+\delta_{j-1}\left(j+\frac{\left|\mathscr{U}^{(j)}-y_{2, j}\right|}{2^{N / 2}}\right)+\frac{\left|\mathscr{U}^{(j)}-y_{2, j}\right|^{3}}{2^{3 N / 2}}\right) \\
& +\left|y_{2, j}-y_{1, j}\right|,
\end{aligned}
$$

provided that

$$
\begin{gathered}
\frac{\tau j^{3 / 2}}{2^{N / 2}} \leqslant c_{59}, \quad\left|\mathscr{U}^{(j)}-y_{2, j}\right| \leqslant \frac{c_{59} \cdot 2^{N}}{j \tau} \\
\frac{\max \left\{\left|\mathscr{U}^{j-1}\right|,\left|\mathscr{V}^{j-1}\right|\right\}}{2^{N / 2}} \leqslant \frac{c_{59} \cdot 2^{N / 2}}{j^{3 / 2} \tau}
\end{gathered}
$$

where

$$
\begin{gathered}
\left|y_{2, j}\right| \leqslant c \tau \frac{\left\|\mathscr{U}^{j-1}\right\|^{2}}{2^{N}}, \quad\left|y_{2, j}-y_{1, j}\right| \leqslant c\left(\tau^{2} 2^{-N / 2} \Gamma_{j-1}^{3}+\tau \Lambda_{j-1} \Gamma_{j-1}\right), \\
\delta_{j-1}=\Gamma_{j-1}^{2}+2^{N / 2} \tau^{-1} \Lambda_{j-1} \\
\Lambda_{j-1}=\frac{\left\|\mathscr{U}^{j-1}-\mathscr{V}^{j-1}\right\|}{2^{N / 2}}, \quad \Gamma_{j-1}=\frac{\max \left\{\left\|\mathscr{U}^{j-1}\right\|,\left\|\mathscr{V}^{j-1}\right\|\right\}}{2^{N / 2}}
\end{gathered}
$$


Below we shall use that, obviously,

$$
\begin{gathered}
j \cdot 2^{-N}\left\|\mathscr{U}^{j-1}\right\|^{2} \leqslant j^{2}+j^{1 / 2} \cdot 2^{-3 N / 2}\left\|\mathscr{U}^{j-1}\right\|^{3}, \\
\left\|\mathscr{V}^{j-1}\right\| \leqslant\left\|\mathscr{U}^{j-1}\right\|+\left\|\mathscr{U}^{j-1}-\mathscr{V}^{j-1}\right\| .
\end{gathered}
$$

Using (8.9) and (9.3)-(9.9), we see that one can choose $c_{60}$ to be so large and $c_{61}$ to be so small that, for $1 \leqslant j \leqslant d$,

$$
\left|\mathscr{U}^{(j)}-\mathscr{V}^{(j)}\right| \leqslant \frac{c_{60} \tau^{2}}{2^{N / 2}}\left(d^{2}+\frac{d^{1 / 2}\left\|\mathscr{U}^{j}\right\|^{3}}{2^{3 N / 2}}\right)+\frac{1}{2}\left|\mathscr{U}^{j-1}-\mathscr{V}^{j-1}\right|
$$

provided that

$$
\begin{aligned}
& \frac{\tau^{2} d^{3} p}{2^{N-p}} \leqslant c_{61}, \quad\left|\mathscr{U}^{(j)}\right| \leqslant \frac{c_{61} \cdot 2^{N}}{d \tau}, \\
& \frac{\max \left\{\left|\mathscr{U}^{j-1}\right|,\left|\mathscr{V}^{j-1}\right|\right\}}{2^{N / 2}} \leqslant \frac{c_{61} \cdot 2^{N / 2}}{d^{3 / 2} \tau},
\end{aligned}
$$

where $\left|\mathscr{U}^{0}-\mathscr{V}^{0}\right|=\left|\mathscr{V}^{0}\right| \stackrel{\text { def }}{=} 0$.

Using the induction with respect to $j$, we shall prove that, for $j=$ $1, \ldots, d$,

$$
\begin{gathered}
\left|\mathscr{V}^{(j-1)}\right| \leqslant \frac{c_{61} \cdot 2^{N}}{d^{3 / 2} \tau} \\
\left|\mathscr{U}^{(j)}-\mathscr{V}^{(j)}\right| \leqslant \frac{c_{62} \tau^{2}}{2 \cdot 2^{N / 2}}\left(d^{2}+\frac{d^{1 / 2}\left\|\mathscr{U}^{j}\right\|^{3}}{2^{3 N / 2}}\right)+\frac{1}{2}\left|\mathscr{U}^{j-1}-\mathscr{V}^{j-1}\right|
\end{gathered}
$$

provided that

$$
|\mathscr{U}| \leqslant \frac{c_{63} \cdot 2^{N}}{d^{3 / 2} \tau}, \quad \frac{\tau^{2} d^{3} p}{2^{N-p}} \leqslant c_{64}
$$

where

$$
c_{62}=2 c_{60}, \quad c_{63}=\min \left\{\frac{c_{61}}{6}, \frac{1}{\sqrt{c_{62}}}\right\}, \quad c_{64}=\min \left\{c_{61},\left(\frac{c_{63}}{c_{62}}\right)^{2 / 3}\right\} .
$$

It is clear that (9.15) implies

$$
c_{65} \stackrel{\text { def }}{=} c_{63}+\frac{c_{62}}{2}\left(c_{64}^{3 / 2}+c_{63}^{3}\right)+\frac{c_{63}}{2}+\frac{c_{61}}{2} \leqslant c_{61} .
$$

Inequality (9.13) for $j=1$ follows directly from (9.10), (9.15), since the conditions (9.11) are in this case the consequences of (9.14) and (9.15). Inequality (9.12) for $j=1$ is valid automatically.

Suppose now that the inequalities (9.12), (9.13) are proved for $j=1, \ldots, s-1,2 \leqslant s \leqslant d$. We shall prove that these inequalities are valid 
for $j=s$. Using (8.9), (9.12) (with $j=1, \ldots, s-1$ ), (9.13) (with $j=s-1$ ) and (9.14)-(9.16), we obtain

$$
\left|\mathscr{V}^{(s-1)}\right| \leqslant\left|\mathscr{U}^{(s-1)}\right|+\left|\mathscr{U}^{(s-1)}-\mathscr{V}^{(s-1)}\right| \leqslant \frac{c_{65} \cdot 2^{N}}{d^{3 / 2} \tau} \leqslant \frac{c_{61} \cdot 2^{N}}{d^{3 / 2} \tau} .
$$

Furthermore, it is clear that the inequality (9.13) for $j=s$ can be easily derived from (9.10), (9.15) since the validity of (9.11) follows in this case from (9.12) (with $j=1, \ldots, s-1),(9.14),(9.15),(9.17),(5.17),(5.18)$ and (8.9). This completes the proof of $(9.12),(9.13)$ for $j=1, \ldots, d$.

Taking into account (5.13), (5.17), (6.23) and (8.9) we see that (9.13) (considered for all $j=1, \ldots, d$ ) implies

$$
|\mathscr{U}-\mathscr{V}| \leqslant \frac{c_{62} \tau^{2}}{2^{N / 2}}\left(d^{2}+\frac{d^{1 / 2}\|\mathscr{U}\|^{3}}{2^{3 N / 2}}\right)
$$

and

$$
|\mathscr{U}| \leqslant\|\mathscr{U}\| \leqslant C_{1}^{-1}\left\|U_{N, 1}\right\|, \quad\left\|U_{N, 1}-V_{N, 1}\right\| \leqslant C_{2} d^{1 / 2}|\mathscr{U}-\mathscr{V}| .
$$

This completes the proof of assertion a) provided that $c_{58}$ is chosen to be sufficiently large and $c_{57}$ sufficiently small.

Let us prove now the assertion b). By condition B (see (6.28) and (6.29)), the coordinates $\mathbf{U}_{n, k}^{(1)}, \ldots, \mathbf{U}_{n, k}^{(2 d)}$ of the random vectors $\mathbf{U}_{n, k}=$ $\mathbb{A}_{n, k} \widetilde{\mathbf{U}}_{n, k}$ and $\mathbf{U}_{n, k}^{j}=\overline{\mathbb{P}}_{j}\left(\mathbf{U}_{n, k}\right), j=1, \ldots, 2 d$, are noncorrelated and have mean zero and the same variance $2^{n}$. The same can be said about the coordinates $\mathbf{V}_{n, k}^{(1)}, \ldots, \mathbf{V}_{n, k}^{(2 d)}$ of the vectors $\mathbf{V}_{n, k}=\mathbb{A}_{n, k} \tilde{\mathbf{V}}_{n, k}$ and $\mathbf{V}_{n, k}^{j}=\overline{\mathbb{P}}_{j}\left(\mathbf{V}_{n, k}\right)$. By Lemma 7.4,

$$
\mathscr{L}\left(\mathbf{U}_{n, k}^{j}\right), \mathscr{L}\left(\mathbf{V}_{n, k}^{j}\right) \in \mathscr{A}_{j}^{*}\left(c_{30} \tau, 4,4\right), \quad j=1, \ldots, 2 d,
$$

provided that $\tau \geqslant 1$ and $\tau^{2} d^{3}(p+1) / 2^{n-p} \leqslant c_{29}$. Thus, the distributions $\mathscr{L}\left(\mathbf{U}_{n, k}^{j}\right), \mathscr{L}\left(\mathbf{V}_{n, k}^{j}\right), j=d+1, \ldots, 2 d$, satisfy in the $j$-dimensional situation the conditions of Lemma 3.3 with $\operatorname{cov} \xi_{k}=\operatorname{cov} \mathbf{U}_{n, k}^{j}=2^{n} \mathbb{I}_{j}$, $\mathbb{B}=\operatorname{cov} \mathbf{U}_{n, k}^{j-1}=2^{n} \mathbb{I}_{j-1}$ and with the change of $\tau$ by $c_{30} \tau$ provided that $c_{57} \leqslant c_{28} / 2$.

Taking into account (5.20) and applying Lemmas 3.3, 8.2 in the $j$-dimensional case, $j=d+1, \ldots, 2 d$, we obtain that

$$
\begin{aligned}
\left|\mathbf{U}_{n, k}^{(j)}-\mathbf{V}_{n, k}^{(j)}\right| \leqslant & \frac{c \tau^{2}}{2^{n / 2}}\left(j^{2}+\delta_{j-1}\left(j+\frac{\left|\mathbf{U}_{n, k}^{(j)}-y_{2, j}\right|}{2^{n / 2}}\right)+\frac{\left|\mathbf{U}_{n, k}^{(j)}-y_{2, j}\right|^{3}}{2^{3 n / 2}}\right) \\
& +\left|y_{2, j}-y_{1, j}\right|,
\end{aligned}
$$

provided that

$$
\frac{\tau j^{3 / 2}}{2^{n / 2}} \leqslant c_{66}, \quad\left|\mathbf{U}_{n, k}^{(j)}-y_{2, j}\right| \leqslant \frac{c_{66} \cdot 2^{n}}{j \tau}, \quad \frac{\max \left\{\left|\mathbf{U}_{n, k}^{j-1}\right|,\left|\mathbf{V}_{n, k}^{j-1}\right|\right\}}{2^{n / 2}} \leqslant \frac{c_{66} \cdot 2^{n / 2}}{j^{3 / 2} \tau}
$$


where

$$
\begin{gathered}
\left|y_{2, j}\right| \leqslant c \tau \frac{\left\|\mathbf{U}_{n, k}^{j-1}\right\|^{2}}{2^{n}}, \quad\left|y_{2, j}-y_{1, j}\right| \leqslant c\left(\tau^{2} 2^{-n / 2} \Gamma_{j-1}^{3}+\tau \Lambda_{j-1} \Gamma_{j-1}\right), \\
\delta_{j-1}=\Gamma_{j-1}^{2}+2^{n / 2} \tau^{-1} \Lambda_{j-1}, \\
\Lambda_{j-1}=\frac{\left\|\mathbf{U}_{n, k}^{j-1}-\mathbf{V}_{n, k}^{j-1}\right\|}{2^{n / 2}}, \quad \Gamma_{j-1}=\frac{\max \left\{\left\|\mathbf{U}_{n, k}^{j-1}\right\|,\left\|\mathbf{V}_{n, k}^{j-1}\right\|\right\}}{2^{n / 2}} .
\end{gathered}
$$

Obviously,

$$
\begin{gathered}
j \cdot 2^{-n}\left\|\mathbf{U}_{n, k}^{j-1}\right\|^{2} \leqslant j^{2}+j^{1 / 2} \cdot 2^{-3 n / 2}\left\|\mathbf{U}_{n, k}^{j-1}\right\|^{3}, \\
\left\|\mathbf{V}_{n, k}^{j-1}\right\| \leqslant\left\|\mathbf{U}_{n, k}^{j-1}\right\|+\left\|\mathbf{U}_{n, k}^{j-1}-\mathbf{V}_{n, k}^{j-1}\right\| .
\end{gathered}
$$

Using (8.16) and (9.18)-(9.23), we see that one can choose $c_{67}$ to be so large and $c_{68}$ to be so small that, for $j=d+1, \ldots, 2 d$,

$$
\left|\mathbf{U}_{n, k}^{(j)}-\mathbf{V}_{n, k}^{(j)}\right| \leqslant \frac{c_{67} \tau^{2}}{2^{n / 2}}\left(d^{2}+\frac{d^{1 / 2}\left\|\mathbf{U}_{n, k}^{j}\right\|^{3}}{2^{3 n / 2}}\right)+\frac{c_{69}}{\sqrt{d}}\left|\mathbf{U}_{n, k}^{j-1}-\mathbf{V}_{n, k}^{j-1}\right|,
$$

where

$$
c_{69}=\frac{\min \left\{C_{1} c_{17}^{-1}, 1\right\}}{16},
$$

provided that

$$
\frac{\tau^{2} d^{3} p}{2^{n-p}} \leqslant c_{68}, \quad\left|\mathbf{U}_{n, k}^{(j)}\right| \leqslant \frac{c_{68} \cdot 2^{n}}{d \tau}, \quad \frac{\max \left\{\left|\mathbf{U}_{n, k}^{j-1}\right|,\left|\mathbf{V}_{n, k}^{j-1}\right|\right\}}{2^{n / 2}} \leqslant \frac{c_{68} \cdot 2^{n / 2}}{d^{3 / 2} \tau} .
$$

Using the induction with respect to $j$, we shall prove that, for $j=$ $d+1, \ldots, 2 d$,

$$
\begin{aligned}
\left|\mathbf{V}_{n, k}^{(j-1)}\right| \leqslant & \frac{c_{68} \cdot 2^{n}}{d^{3 / 2} \tau} \\
\left|\mathbf{U}_{n, k}^{(j)}-\mathbf{V}_{n, k}^{(j)}\right| \leqslant & \frac{c_{70} \tau^{2}}{2 \cdot 2^{n / 2}}\left(d^{2}+\frac{d^{1 / 2}\left\|\mathbf{U}_{n, k}^{j}\right\|^{3}}{2^{3 n / 2}}\right) \\
& +\frac{c_{69}}{\sqrt{d}}\left|\mathbf{U}_{n, k}^{j-1}-\mathbf{V}_{n, k}^{j-1}\right|
\end{aligned}
$$

provided that

$$
\max \left\{\left|\mathbf{U}_{n, k}\right|,\left|\mathscr{V}_{n, k}\right|\right\} \leqslant \frac{c_{71} \cdot 2^{n}}{d^{3 / 2} \tau}, \quad \frac{\tau^{2} d^{3} p}{2^{n-p}} \leqslant c_{72}
$$

where

$$
c_{70}=2 c_{67}, \quad c_{71}=\min \left\{\frac{c_{68}}{4}, \frac{1}{\sqrt{c_{70}}}\right\}, \quad c_{72}=\min \left\{c_{68},\left(\frac{c_{71}}{c_{70}}\right)^{2 / 3}\right\} .
$$


It is clear that (9.30) implies

$$
c_{73} \stackrel{\text { def }}{=} c_{71}+\frac{c_{70}}{2}\left(c_{72}^{3 / 2}+c_{71}^{3}\right)+\frac{c_{71}}{8}+\frac{c_{68}}{16} \leqslant c_{68} .
$$

Inequality (9.27) for $j=d+1$ immediately follows from (9.29) and (9.30) since $\mathbf{V}_{n, k}^{(d)}=\mathbb{P}_{d} \mathscr{V}_{n, k}$ (see (5.9), (6.7) and (6.8)). Inequality (9.28) for $j=d+1$ follows directly from (9.24) and (9.30), since the conditions (9.26) are in this case the consequences of (5.21), (6.8), (9.29) and (9.30).

Assume now that the inequalities (9.27), (9.28) are proved for $j=d+$ $1, \ldots, s-1, d+2 \leqslant s \leqslant 2 d$. We shall prove that these inequalities are valid for $j=s$. Using (5.21), (6.8), (8.16), (9.25), (9.27) (with $j=d+1, \ldots, s-1$ ), (9.28) (with $j=s-1$ ) and (9.29)-(9.31), we obtain

$$
\left|\mathbf{V}_{n, k}^{(s-1)}\right| \leqslant\left|\mathbf{U}_{n, k}^{(s-1)}\right|+\left|\mathbf{U}_{n, k}^{(s-1)}-\mathbf{V}_{n, k}^{(s-1)}\right| \leqslant \frac{c_{73} \cdot 2^{n}}{d^{3 / 2} \tau} \leqslant \frac{c_{68} \cdot 2^{n}}{d^{3 / 2} \tau}
$$

Furthermore, it is clear that the inequality (9.28) for $j=s$ can easily be derived from (9.24), (9.30) since the validity of (9.26) follows in this case from $(5.21),(8.16),(9.27)$ (with $j=d+1, \ldots, s-1),(9.29),(9.30)$ and (9.32). This completes the proof of (9.27), (9.28) for $j=d+1, \ldots, 2 d$.

Taking into account $(5.21),(5.24),(6.4),(6.5),(6.7),(6.8),(6.23)$ and (9.25), we see that (9.28) (considered for all $j=d+1, \ldots, 2 d)$ implies

$$
\begin{aligned}
\left|\widetilde{\mathscr{U}}_{n, k}-\widetilde{\mathscr{V}}_{n, k}\right| \leqslant & \frac{c_{70} \tau^{2}}{2 \cdot 2^{n / 2}}\left(d^{2}+\frac{d^{1 / 2}\left\|\mathbf{U}_{n, k}\right\|^{3}}{2^{3 n / 2}}\right)+\frac{1}{16}\left|\widetilde{\mathscr{U}}_{n, k}-\widetilde{\mathscr{V}}_{n, k}\right| \\
& +\frac{C_{1} c_{17}^{-1}}{16 \sqrt{d}}\left|\mathscr{U}_{n, k}-\mathscr{V}_{n, k}\right|
\end{aligned}
$$

and $\left|\mathscr{V}_{n, k}\right| \leqslant C_{1}^{-1}\left\|V_{n, k},\left|\mathscr{U}_{n, k}-\mathscr{V}_{n, k}\right| \leqslant C_{1}^{-1}\right\| U_{n, k}-V_{n, k} \|$. Together with trivial bound $\|x\| \leqslant \sqrt{d}|x|, x \in \mathbb{R}^{d}$, this completes the proof of assertion $\mathrm{b}$ ) provided that $c_{58}$ is chosen to be sufficiently large and $c_{57}$ sufficiently small.

10. Proof of Theorem 1.5. Let $u, v, w$ be arbitrary numbers satisfying the relations

$$
\frac{5}{8} \leqslant v<\frac{2}{3}, \quad u=1-v, \quad w=2-3 v .
$$

It is clear that

$$
\frac{1}{3}<u \leqslant \frac{3}{8}, \quad 0<w \leqslant \frac{1}{8}, \quad 3 u-w=1 .
$$

Below the symbols $a_{5}, \ldots, a_{11}$ will be used for denoting positive quantities depending only on $v$ (and, of course, on $C_{1}$ and $C_{2}$ ). The letter $a$ will denote different positive quantities depending only on $v, C_{1}$ and $C_{2}$ when we do not 
need to fix their numerical values. It is clear that, if $v, C_{1}$ and $C_{2}$ are fixed, $a, a_{5}, \ldots, a_{11}$ are absolute constants. Write

$$
\begin{aligned}
& c_{74}=\min \left\{c_{57}, 1\right\}, \\
& a_{5}=\min \left\{\frac{c_{74}}{4}, \frac{c_{74} c_{16}}{4}, \frac{1}{\left(18 c_{58} c_{17}\right)^{1 / 2}},\right. \\
& \\
&\left.\quad \frac{c_{16}^{3 / 2}}{\left(65 c_{58} c_{17}\right)^{1 / 2}}, \frac{1-2^{-w}}{2}, C_{2}^{2}\right\}, \\
& a_{6}=\min \left\{c_{57}, a_{5}^{2}, 1\right\} .
\end{aligned}
$$

Recall that (see Lemma 6.1)

$$
c_{17} \geqslant 1
$$

Let

$$
\tau \geqslant 1, \quad \lambda \geqslant \max \left\{C_{2}, 1\right\} \sqrt{d}
$$

Define $p$ as the minimal integer satisfying the inequalities

$$
p \geqslant 4, \quad \frac{a_{5} \cdot 2^{v p}}{d^{3 / 2} \tau} \geqslant 4 \lambda
$$

Denote by $M$ the maximal integer such that

$$
\frac{\tau^{2} d^{3} p}{2^{M-p}} \geqslant a_{6}
$$

Let $N$ be a positive integer such that

$$
\frac{\tau^{2} d^{3} p}{2^{N-p}}<a_{6}, \quad \frac{N-M}{p} \text { is integer. }
$$

Obviously, the relations (10.3), (10.5)-(10.8) imply

$$
N>M \geqslant p, \quad N \geqslant 2 p \text {. }
$$

Let $\tilde{\xi}_{1}, \ldots, \tilde{\xi}_{2^{N}}$ be random vectors with distributions $\mathscr{L}\left(\tilde{\xi}_{k}\right) \in \mathscr{A}_{d}(\tau)$, $\mathbf{E} \tilde{\xi}_{k}=0, \operatorname{cov} \tilde{\xi}_{k}=\mathbb{B}_{k}, k=1, \ldots, 2^{N}$. Assume that the covariance operators $\mathbb{B}_{k}$ satisfy the conditions (6.2). Let $\widetilde{\eta}_{k}$ be random vectors which have the same moments of the first three orders as the vectors $\tilde{\xi}_{k}$, and

$$
\mathscr{L}\left(\widetilde{\eta}_{k}\right) \in \mathscr{A}_{d}(\tau), \quad \mathbf{P}\left\{\left\|\widetilde{\eta}_{k}\right\| \leqslant \lambda\right\}=1, \quad k=1, \ldots, 2^{N}
$$

Suppose that the independent random vectors $\tilde{Y}_{k}, k=1, \ldots, 2^{N}$, with

$$
\mathscr{L}\left(\tilde{Y}_{k}\right)=\Psi\left(\mathscr{L}\left(\widetilde{\eta}_{k}\right)\right)
$$


are already constructed (the distributions $\Psi(\cdot)$ were defined in Section 7 ). According to Lemma $7.2, \widetilde{Y}_{k}$ and $\tilde{\xi}_{k}$ have the same moments of the first three orders. Our aim is to construct independent random vectors $\widetilde{X}_{k}$, $k=1, \ldots, 2^{N}$, with $\mathscr{L}\left(\tilde{X}_{k}\right)=\mathscr{L}\left(\tilde{\xi}_{k}\right)$ so that the sequential sums of $\tilde{X}_{k}$ and $\widetilde{Y}_{k}$ would be close with large probability. To this end we define the random vectors

$$
\left\{Y_{k}^{[N-l p]}, Z_{(k-1) \cdot 2^{l_{p}}+1}^{N-l p}, 1 \leqslant k \leqslant 2^{N-l p}, 0 \leqslant l \leqslant \frac{N-M-p}{p}\right\}
$$

with the help of a recurrent procedure described below. In one-dimensional case a very similar procedure was used by Sakhanenko [18]. Note also that the arguments of this section are close to those in [29, Section 13] and [31, Section 8].

At first, we set $Y_{k}^{[N]}=\tilde{Y}_{k}, k=1, \ldots, 2^{N}$. After this, using the dyadic procedure described in Sections 5-7 and 9 and satisfying Condition $\mathbf{B}$, we define the independent random vectors $Z_{k}^{[N]}, k=1, \ldots, 2^{N}$, with distributions $\mathscr{L}\left(Z_{r}^{[N]}\right)=\Psi\left(\mathscr{L}\left(\widetilde{\eta}_{r}\right)\right)$ for $r=(m-1) \cdot 2^{p}+1, m=1, \ldots, 2^{N-p}$. For $r=1, \ldots, 2^{N}, r \neq(m-1) \cdot 2^{p}+1, m=1, \ldots, 2^{N-p}$, we take $\mathscr{L}\left(Z_{r}^{[N]}\right)=\mathscr{L}\left(\tilde{\xi}_{r}\right)$ and define $\tilde{X}_{r}=Z_{r}^{[N]}$. Thus, the amount of vectors $\widetilde{X}_{k}$, which are not constructed, is decreased in $2^{p}$ times. If $N=M+p$, the first step of applying this procedure is simultaneously the last one, since the random vectors (10.12) with $l=(N-M-p) / p=0$ are already constructed.

Suppose now that $N>M+p$. Then we repeat this procedure, working only with vectors which have the distributions $\Psi\left(\mathscr{L}\left(\widetilde{\eta}_{r}\right)\right)$ and diminishing their number after each step in $2^{p}$ times.

Namely, if the vectors $\left\{Y_{k}^{[N-l p+p]}, Z_{(k-1) \cdot 2^{l p-p+1}}^{[N-l p+p]}, 1 \leqslant k \leqslant 2^{N-l p+p}\right\}$ are already defined for an integer $l$ and $1 \leqslant l \leqslant(N-M-p) / p$, we set $Y_{k}^{[N-l p]}=Z_{(k-1) \cdot 2^{l p}+1}^{[N-l p+p]}, \quad k=1, \ldots, 2^{N-l p}$, and, using again the dyadic procedure described in Sections $5-7$ and 9 and satisfying Condition $\mathbf{B}$ (with $N-l p$ instead of $N$ ), construct the independent random vectors $Z_{(k-1) \cdot 2^{l_{p}+1}}^{[N-l]}, k=1, \ldots, 2^{N-l p}$, with distributions written out below. If $r=(m-1) \cdot 2^{l p+p}+1, m=1, \ldots, 2^{N-l p-p}$, we choose $\mathscr{L}\left(Z_{r}^{[N-l p]}\right)=\Psi\left(\mathscr{L}\left(\widetilde{\eta}_{r}\right)\right)$. In the case $r=(s-1) \cdot 2^{l p}+1, s=1, \ldots, 2^{N-l p}$, and $r \neq(m-1) \cdot 2^{l p+p}+1$, $m=1, \ldots, 2^{N-l p-p}$, we take $\mathscr{L}\left(Z_{r}^{[N-l p]}\right)=\mathscr{L}\left(\tilde{\xi}_{r}\right)$ and define $\tilde{X}_{r}=Z_{r}^{[N-l p]}$.

It is easy to see that for each $l, 0 \leqslant l \leqslant(N-M-p) / p$, the dyadic scheme satisfies Conditions $\mathbf{A}(\tau), \mathbf{B}, \mathbf{D}_{\Psi}$ and $\mathbf{C}_{\Psi}(p)$ (with $N$ - $l p$ instead of $N$ ). In the sequel this allows us to apply Lemma 9.1.

After the last step (with $l=(N-M-p) / p$ ) all independent random vectors $\widetilde{X}_{k}, k=1, \ldots, 2^{N}$, are constructed except $\widetilde{X}_{r}$ having indices of the form $r=(m-1) \cdot 2^{N-M}+1, m=1, \ldots, 2^{M}$. Instead of this we have constructed the vectors with distributions $\mathscr{L}\left(Z_{r}^{[M+p]}\right)=\Psi\left(\mathscr{L}\left(\widetilde{\eta}_{r}\right)\right)$ for $r=$ $(m-1) \cdot 2^{N-M}+1, m=1, \ldots, 2^{M}$. Together with $\widetilde{X}_{r}$ these vectors form a collection of jointly independent vectors. This can easily be verified by 
induction, if we take into account that, in each step, the new collection of independent vectors is constructed as a function of the vectors which are independent of all already constructed vectors $\tilde{X}_{r}$.

Now we can add the missing elements to the sequence of already constructed $\widetilde{X}_{r}$. For this we define the random vectors $Y_{k}^{*}=Y_{k}^{[M]}=$ $Z_{(k-1) \cdot 2^{N-M}+1}^{[M+p]}, k=1, \ldots, 2^{M}$, and, using again the procedure described in Sections $5-7$, construct the independent random vectors $X_{k}^{*}, k=1, \ldots, 2^{M}$, with $\mathscr{L}\left(X_{k}^{*}\right)=\mathscr{L}\left(\tilde{\xi}_{(k-1) \cdot 2^{N-M}+1}\right)$. Then we set $\tilde{X}_{(k-1) \cdot 2^{N-M}+1}=X_{k}^{*}$. It is clear that the vectors $X_{k}^{*}$ are jointly independent and they do not depend on $\widetilde{X}_{r}$ constructed earlier, since, by construction, $X_{k}^{*}$ are functions of $Y_{k}^{*}$. This completes the procedure of constructing $\widetilde{X}_{r}, r=1, \ldots, 2^{N}$.

By analogy with arguments in [18], we shall prove that the procedure described above gives good approximation, using an induction with respect to $N$. It is clear that the procedure of constructing the independent random vectors $X_{*, k}=\widetilde{X}_{(k-1) \cdot 2^{p}+1}, k=1, \ldots, 2^{N-p}$, coincides with the procedure of constructing $\tilde{X}_{k}, k=1, \ldots, 2^{N}$. The only difference is in the change of $N$ by $N-p$. Thus, below we shall assume that we have suitable approximation in the case when we construct $2^{N-p}$ summands and prove the analogous statement in the case of $2^{N}$ summands.

We shall use the notation which was already used in Sections 5-7 and 9 with $Y_{k}=Y_{k}^{[N]}, X_{k}=Z_{k}^{[N]}, k=1, \ldots, 2^{N}$. Let $j$ be an integer satisfying $1 \leqslant j \leqslant 2^{N}$. Write $S=S_{j}, T=T_{j}$ (see (5.7), (5.23)). Sometimes we shall omit for simplicity the index $j$, keeping in mind that it is fixed. By virtue of (6.2), (10.5), (10.10), (10.11), by construction and by the definition of distributions $\Psi(\cdot)$ (see $(7.12)-(7.14)$ ) we have

$$
\mathbf{P}\left\{\left\|Y_{k}\right\| \leqslant 4 \lambda\right\}=1, \quad k=1, \ldots, 2^{N} .
$$

It is clear that from (5.7), (10.13) it follows that

$$
\mathbf{P}\left\{\|T\| \leqslant 4 \lambda \cdot 2^{N}\right\}=1 .
$$

For fixed integer $s, 0 \leqslant s \leqslant N$, define the integer $k_{s}=k_{s}(j)$ by the relation

$$
\left(k_{s}-1\right) \cdot 2^{s}<j \leqslant k_{s} \cdot 2^{s} .
$$

Now we can represent the sums $S$ and $T$ in the form

$$
S=S^{(s)}-S^{\{s\}}, \quad T=T^{(s)}-T^{\{s\}},
$$

where

$$
\begin{aligned}
& S^{(s)}=S_{k_{s} \cdot 2^{s}}, \quad S^{\{s\}}=S^{(s)}-S, \\
& T^{(s)}=T_{k_{s} \cdot 2^{s}}, \quad T^{\{s\}}=T^{(s)}-T .
\end{aligned}
$$


According to Lemma 6.1,

$$
\left\|S^{(s)}-T^{(s)}\right\| \leqslant c_{17}\left\|U_{N, 1}-V_{N, 1}\right\|+c_{17} \sum_{n=s+1}^{N}\left\|\widetilde{\mathscr{U}}^{(n)}-\widetilde{\mathscr{V}}^{(n)}\right\|,
$$

where $\widetilde{\mathscr{U}}^{(n)}=\widetilde{\mathscr{U}}_{n, l_{n, j}}, \widetilde{\mathscr{V}}^{(n)}=\widetilde{\mathscr{V}}_{n, l_{n, j}},\left(l_{n, j}-1\right) \cdot 2^{n}<j \leqslant l_{n, j} \cdot 2^{n}($ see $(5.32))$. Write as well, by analogy with the proof of Theorem 8.1,

$$
\begin{array}{lll}
U^{(n)}=U_{n, l_{n, j}}, & V^{(n)}=V_{n, l_{n, j}}, & U_{(n)}=U_{n, \widetilde{l}_{n, j}} \\
\mathbf{U}^{(n)}=\mathbf{U}_{n, l_{n, j}}, & \tilde{\mathbf{U}}^{(n)}=\tilde{\mathbf{U}}_{n, l_{n, j}}, & \mathbb{A}^{(n)}=\mathbb{A}_{n, l_{n, j}},
\end{array}
$$

where

$$
\tilde{l}_{n-1, j}= \begin{cases}2 l_{n, j}, & \text { if } l_{n-1, j}=2 l_{n, j}-1, \\ 2 l_{n, j}-1, & \text { if } l_{n-1, j}=2 l_{n, j}\end{cases}
$$

$0 \leqslant n \leqslant N$. As before, according to Remark $5.2, l_{n, j}$ is the integer such that $U^{(n)}$, the sum over a block of $2^{n}$ summands, contains the summand $X_{j}$ and, consequently, the summand $X_{k_{s} \cdot 2^{s}}$, for $s<n \leqslant N$ (see (10.15)). The sum $U_{(n)}$ does not contain $X_{j}$ and, by (5.24), (6.38), (10.19),

$$
\begin{gathered}
U^{(n+1)}=U^{(n)}+U_{(n)}, \quad\left\|\tilde{\mathbf{U}}^{(n+1)}\right\|^{2}=\left\|U^{(n)}\right\|^{2}+\left\|U_{(n)}\right\|^{2}, \\
\left\|U^{(n+1)}\right\|=\mathbb{A}^{(n+1)} \tilde{\mathbf{U}}^{(n+1)}, \quad\left\|\mathbb{A}^{(n+1)}\right\| \leqslant c_{16}^{-1},
\end{gathered}
$$

$0 \leqslant n<N$. From (10.21) it follows that

$$
\left\|U_{(n)}\right\|=\left\|U^{(n+1)}-U^{(n)}\right\| \leqslant\left\|U^{(n+1)}\right\|+\left\|U^{(n)}\right\|
$$

and, hence,

$$
\begin{aligned}
& \left\|\tilde{\mathbf{U}}^{(n+1)}\right\| \leqslant 2 \max \left\{\left\|U^{(n)}\right\|,\left\|U_{(n)}\right\|\right\} \leqslant 2\left\|U^{(n+1)}\right\|+2\left\|U^{(n)}\right\|, \\
& \left\|\mathbf{U}^{(n+1)}\right\| \leqslant 2 c_{16}^{-1}\left(\left\|U^{(n+1)}\right\|+\left\|U^{(n)}\right\|\right) .
\end{aligned}
$$

It is evident that $U^{(N)}=U_{N, 1}, V^{(N)}=V_{N, 1}$. Thus, from (10.16), (10.18) it follows that

$$
\begin{aligned}
\|S-T\| \leqslant & c_{17}\left\|U^{(N)}-V^{(N)}\right\|+c_{17} \sum_{n=s+1}^{N}\left\|\widetilde{\mathscr{U}}^{(n)}-\widetilde{\mathscr{V}}^{(n)}\right\| \\
& +\left\|S^{\{s\}}\right\|+\left\|T^{\{s\}}\right\| .
\end{aligned}
$$

Introduce the random integer $\mu=\mu_{j}$ by $\mu=N+1$, if at least one of the following inequalities is valid:

$$
\frac{\tau^{2} d^{3} p}{2^{N-p}}>a_{6}, \quad\left\|S^{\{N\}}\right\|>\frac{2 a_{5} \cdot 2^{N}}{d^{3 / 2} \tau}, \quad\left\|U^{(N)}\right\|>\frac{2 a_{5} \cdot 2^{N}}{d^{3 / 2} \tau}
$$


and by

$$
\begin{gathered}
\mu=\min \left\{m: p \leqslant m \leqslant N, \frac{\tau^{2} d^{3} p}{2^{m-p}} \leqslant a_{6} ;\left\|S^{\{n\}}\right\| \leqslant \frac{2 a_{5} \cdot 2^{n}}{d^{3 / 2} \tau}\right. \\
\left.\left\|U^{(n)}\right\| \leqslant \frac{2 a_{5} \cdot 2^{u m+v n}}{d^{3 / 2} \tau}, n=m, \ldots, N\right\}
\end{gathered}
$$

otherwise. Here the numbers $u, v$ are defined by (10.1). Note that the first of inequalities (10.25) is not satisfied in our conditions (see (10.8)). The same can be said about inequalities (10.41), (10.45).

Write

$$
Y_{*, k}=Y_{k}^{[N-p]}=Z_{(k-1) \cdot 2^{p+1}}^{N}, \quad k=1, \ldots, 2^{N-p} .
$$

By construction and by (6.2), (7.12)-(7.14), (10.5), (10.10), (cf. (10.13)), we have

$$
\mathbf{P}\left\{\left\|Y_{*, k}\right\| \leqslant 4 \lambda\right\}=1, \quad k=1, \ldots, 2^{N-p} .
$$

Let $i=i(j)$ be the largest integer such that $(i-1) \cdot 2^{p}+1 \leqslant j$. We shall denote by $V_{*}^{(n)}, n=0, \ldots, N-p$, the sum of a block of $2^{n}$ summands $Y_{*, k}$ which contains the summand $Y_{*, i}$. Let

$$
T_{*}=T_{*, i}=Y_{*, 1}+\cdots+Y_{*, i} .
$$

Recall that, by construction,

$$
\begin{aligned}
& \bigcup_{k=1}^{2^{N-p}}\left\{\left\{\tilde{X}_{r}=Z_{r}^{[N]}, r=(k-1) \cdot 2^{p}+2, \ldots, k \cdot 2^{p}\right\}\right. \\
& \left.\cup\left\{Y_{*, k}=Z_{(k-1) \cdot 2^{p}+1}^{[N]}\right\}\right\}
\end{aligned}
$$

is a collection of random vectors, which are jointly independent. Set

$$
U_{0}^{(n)}=U^{(n)}-V_{*}^{[n-p]}, \quad n=p, \ldots, N .
$$

It can easily be verified that the sum $U_{0}^{(n)}$ consists of the summands of the sum $U^{[n]}$ with indices which can not be represented in the form $r=$ $(k-1) \cdot 2^{p}+1$ with integer $k$. In other words, the sum $U_{0}^{(n)}$ is the sum of contained in $U^{[n]}$ summands $Z_{r}^{[N]}$ which are chosen as $\widetilde{X}_{r}$. For the proof of this fact one should take into account that, by construction, the numbers $j$ and $(i-1) \cdot 2^{p}+1$ are contained in the same block of indices $\{m: m=$ $\left.(i-1) \cdot 2^{p}+1, \ldots, i \cdot 2^{p}\right\}$. Recall that, according to our construction, after the first step, when the independent random vectors (10.30) are constructed, 
we define the rest of the random vectors $\tilde{X}_{r}$ as the (d-dimensional) functions of the random vectors $Y_{*, k}, k=1, \ldots, 2^{N-p}$. Write

$$
\begin{gathered}
X_{*, k}=\tilde{X}_{(k-1) \cdot 2^{p}+1}, \quad k=1, \ldots, 2^{N-p} ; \\
S_{*}=S_{*, i}=X_{*, 1}+\cdots+X_{*, i}, \quad S_{0}=S-T_{*} .
\end{gathered}
$$

By analogy with (10.16), (10.17), for $s=p, \ldots, N$ we can represent the sums $S_{*}, S_{0}$ and $T_{*}$ in the form

$$
S_{0}=S_{0}^{(s)}-S_{0}^{\{s\}}, \quad T_{*}=T_{*}^{(s-p)}-T_{*}^{\{s-p\}},
$$

where

$$
\begin{gathered}
T_{*}^{(s-p)}=T_{*, k_{s} \cdot 2^{s-p}}, \quad T_{*}^{\{s-p\}}=T_{*}^{(s-p)}-T_{*}, \\
S_{0}^{(s)}=S^{(s)}-T_{*}^{(s-p)}, \quad S_{0}^{\{s\}}=S_{0}^{(s)}-S_{0}=S^{\{s\}}-T_{*}^{\{s-p\}},
\end{gathered}
$$

and the numbers $k_{s}$ are defined by (10.15).

From the definition of numbers $i, j, k_{s}, s=0, \ldots, N$, it follows that

$$
\begin{array}{ll}
0 \leqslant k_{s} \cdot 2^{s}-j<2^{s}, & s=0, \ldots, N ; \\
0 \leqslant k_{s} \cdot 2^{s-p}-i<2^{s-p}, & s=p, \ldots, N .
\end{array}
$$

It is easy to see that from (5.7), (10.13), (10.16), (10.17), (10.28), (10.29), (10.34), (10.35) one can derive that, with probability one,

$$
\begin{aligned}
\left\|T^{\{s\}}\right\| & \leqslant 4 \lambda \cdot 2^{s}, \quad s=0, \ldots, N ; \\
\left\|T_{*}^{\{s-p\}}\right\| \leqslant 4 \lambda \cdot 2^{s-p}, & s=p, \ldots, N .
\end{aligned}
$$

Similarly, using (10.28) and the definition of $V_{*}^{(n)}$, we see that

$$
\left\|V_{*}^{(n)}\right\| \leqslant 4 \lambda \cdot 2^{n} \quad \text { a.s., } \quad n=0, \ldots, N-p .
$$

Define also

$$
\begin{aligned}
\widetilde{S} & =\sum_{k=1}^{j} \tilde{X}_{k}, \\
\delta_{j}(N) & =\left\|\sum_{k=1}^{j} \widetilde{Y}_{k}-\sum_{k=1}^{j} \tilde{X}_{k}\right\|=\|T-\widetilde{S}\|, \\
\delta_{i}(N-p) & =\left\|\sum_{k=1}^{i} Y_{*, k}-\sum_{k=1}^{i} X_{*, k}\right\|=\left\|T_{*}-S_{*}\right\|, \\
\psi_{j}(N) & =\left\|\sum_{k=1}^{j} \tilde{Y}_{k}-\sum_{k=1}^{j} Z_{k}^{[N]}\right\|=\|T-S\|
\end{aligned}
$$

(see (5.7), (5.23), (10.29), (10.32)). It is clear that from (10.27), (10.29), (10.30), (10.32), (10.38) and from the definition of $i$ and $S$ it follows that

$$
S=\sum_{k=1}^{j} Z_{k}^{[N]}=S_{0}+T_{*}, \quad \widetilde{S}=S_{0}+S_{*}, \quad S-\widetilde{S}=T_{*}-S_{*} .
$$


It is easy to see that the relations (10.38), (10.39) imply

$$
\delta_{j}(N) \leqslant \psi_{j}(N)+\delta_{i}(N-p) .
$$

Introduce the random integer $\beta_{j}(N)$ by $\beta_{j}(N)=N+1$, if at least one of the following inequalities is valid:

$$
\frac{\tau^{2} d^{3} p}{2^{N-p}}>a_{6}, \quad\left\|V^{(N)}\right\|>\frac{4 a_{5} \cdot 2^{N}}{d^{3 / 2} \tau}
$$

and by

$$
\begin{aligned}
\beta_{j}(N)=\min \left\{m: p \leqslant m \leqslant N, \frac{\tau^{2} d^{3} p}{2^{m-p}} \leqslant a_{6},\right. \\
\left.\left\|V^{(n)}\right\| \leqslant \frac{4 a_{5} \cdot 2^{u m+v n}}{d^{3 / 2} \tau}, n=m, \ldots, N\right\}
\end{aligned}
$$

otherwise.

Similarly, taking into account (10.9), we define the random integer $\beta_{i}(N-p)$ by the equality $\beta_{i}(N-p)=N-p+1$, if at least one of the following inequalities is valid:

$$
\frac{\tau^{2} d^{3} p}{2^{N-2 p}}>a_{6}, \quad\left\|V_{*}^{(N-p)}\right\|>\frac{4 a_{5} \cdot 2^{N-p}}{d^{3 / 2} \tau}
$$

and by

$$
\begin{aligned}
\beta_{i}(N-p)=\min \left\{m: p \leqslant m \leqslant N-p, \frac{\tau^{2} d^{3} p}{2^{m-p}} \leqslant a_{6},\right. \\
\left.\left\|V_{*}^{(n)}\right\| \leqslant \frac{4 a_{5} \cdot 2^{u m+v n}}{d^{3 / 2} \tau}, n=m, \ldots, N-p\right\}
\end{aligned}
$$

otherwise.

Finally, define the random integer $\nu=\nu_{j}$ by $\nu=N+1$, if at least one of the following inequalities is valid:

$$
\frac{\tau^{2} d^{3} p}{2^{N-p}}>a_{6}, \quad\left\|U_{0}^{(N)}\right\|>\frac{a_{5} \cdot 2^{N}}{d^{3 / 2} \tau}, \quad\left\|S_{0}^{\{N\}}\right\|>\frac{a_{5} \cdot 2^{N}}{d^{3 / 2} \tau}
$$

and by

$$
\begin{gathered}
\nu=\min \left\{m: p \leqslant m \leqslant N, \frac{\tau^{2} d^{3} p}{2^{m-p}} \leqslant a_{6} ;\left\|S_{0}^{\{n\}}\right\| \leqslant \frac{a_{5} \cdot 2^{n}}{d^{3 / 2} \tau},\right. \\
\left.\left\|U_{0}^{(n)}\right\| \leqslant \frac{a_{5} \cdot 2^{u m+v n}}{d^{3 / 2} \tau}, n=m, \ldots, N\right\}
\end{gathered}
$$

otherwise.

Put now

$$
\begin{aligned}
& \rho_{j}(N)=5 \lambda \cdot 2^{\beta_{j}(N)}, \quad \rho_{i}(N-p)=5 \lambda \cdot 2^{\beta_{i}(N-p)}, \\
& \gamma_{j}(N)= \begin{cases}10 \lambda \cdot 2^{\nu}, & \text { if } \nu \leqslant N, \\
U+22 \lambda \cdot 2^{N}, & \text { if } \nu=N+1,\end{cases}
\end{aligned}
$$


where

$$
U=\left\|U_{0}^{(N)}\right\|+\left\|S_{0}^{\{N\}}\right\|
$$

Lemma 10.1. The following inequalities are valid:

$$
\delta_{j}(N)+\rho_{j}(N) \leqslant \delta_{i}(N-p)+\rho_{i}(N-p)+\gamma_{j}(N)
$$

and, for each $\varepsilon>0$,

$$
\begin{aligned}
& \mathbf{E} \exp \left(\varepsilon\left(\delta_{j}(N)+\rho_{j}(N)\right)\right) \\
& \quad \leqslant \mathbf{E} \exp \left(\varepsilon\left(\delta_{i}(N-p)+\rho_{i}(N-p)\right)\right) \mathbf{E} \exp \left(\varepsilon \gamma_{j}(N)\right) .
\end{aligned}
$$

P r o o f. Assume that $\nu \leqslant N$ and, taking into account (10.9), define the integer valued random variables

$$
\beta=\beta_{i}(N-p)-1 \leqslant N-p, \quad \varkappa=\max \{\beta, \nu\} \leqslant N .
$$

The relations (10.1), (10.6), (10.36) imply

$$
\left\|T_{*}^{\{s-p\}}\right\| \leqslant \frac{a_{5} \cdot 2^{s}}{d^{3 / 2} \tau} \quad \text { a.s., } \quad s=p, \ldots, N .
$$

Using (10.34), (10.46), (10.52), (10.53), we obtain

$$
\left\|S^{\{n\}}\right\| \leqslant\left\|S_{0}^{\{n\}}\right\|+\left\|T_{*}^{\{n-p\}}\right\| \leqslant \frac{2 a_{5} \cdot 2^{n}}{d^{3 / 2} \tau}, \quad n=\varkappa, \ldots, N,
$$

(here and below we use that (10.46), (10.52) imply the inequality $\varkappa \geqslant p$ ). By virtue of (10.1), (10.6), (10.37), (10.52), for $\beta \leqslant n \leqslant \beta+p, n \geqslant p$, we have

$$
\left\|V_{*}^{(n-p)}\right\| \leqslant 4 \lambda \cdot 2^{n-p} \leqslant \frac{a_{5} \cdot 2^{u \beta+v n}}{d^{3 / 2} \tau} \leqslant \frac{a_{5} \cdot 2^{u \varkappa+v n}}{d^{3 / 2} \tau} .
$$

If $\beta+p+1 \leqslant n \leqslant N$, then, applying (10.1), (10.2), (10.6), (10.44), (10.52), we obtain

$$
\left\|V_{*}^{(n-p)}\right\| \leqslant \frac{4 a_{5} \cdot 2^{u(\beta+1)+v(n-p)}}{d^{3 / 2} \tau} \leqslant \frac{a_{5} \cdot 2^{u \varkappa+v n}}{d^{3 / 2} \tau}
$$

(we use that the inequalities $p \geqslant 4,(10.1),(10.2)$ imply $2^{v p} \geqslant 2^{5 p / 8}>4 \cdot 2^{3 / 8} \geqslant$ $\left.4 \cdot 2^{u}\right)$. From $(10.31),(10.46),(10.52),(10.55),(10.56)$ it follows that

$$
\begin{aligned}
\left\|U^{(n)}\right\| & \leqslant\left\|U_{0}^{(n)}\right\|+\left\|V_{*}^{(n-p)}\right\| \leqslant \frac{a_{5} \cdot 2^{u \varkappa+v n}}{d^{3 / 2} \tau}+\frac{a_{5} \cdot 2^{u \varkappa+v n}}{d^{3 / 2} \tau} \\
& =\frac{2 a_{5} \cdot 2^{u \varkappa+v n}}{d^{3 / 2} \tau}, \quad n=\varkappa, \ldots, N .
\end{aligned}
$$

Taking into account $(10.26),(10.46),(10.52),(10.54),(10.57)$, we see that

$$
\mu \leqslant \varkappa \leqslant N \text {. }
$$


Suppose now that an integer $n$ satisfies the inequalities

$$
\mu \leqslant n \leqslant N .
$$

Then, taking into account (10.1)-(10.3), (10.6), (10.26), (10.59), we see that

$$
\tau^{2} d^{3} \leqslant a_{6} \cdot 2^{\mu} \leqslant a_{5}^{2} \cdot 2^{2 u \mu+(2 v-1) n} \leqslant a_{5}^{2} \cdot 2^{2 u \mu+(2 v-1) N} .
$$

Using (10.1), (10.3), (10.26), (10.59), we obtain the inequalities

$$
\left\|U^{(n)}\right\| \leqslant \frac{2 a_{5} \cdot 2^{u \mu+v n}}{d^{3 / 2} \tau} \leqslant \frac{c_{74} \cdot 2^{n}}{d^{3 / 2} \tau} \leqslant \frac{c_{57} \cdot 2^{n}}{d^{3 / 2} \tau} .
$$

Taking here $n=N$ and using (10.1)-(10.4), (10.19), (10.26), (10.59)-(10.61) and Lemma 9.1 a), we obtain

$$
\begin{aligned}
\left\|U^{(N)}-V^{(N)}\right\| & \leqslant c_{58} \tau^{2} \cdot 2^{-N / 2}\left(d^{5 / 2}+d \cdot 2^{-3 N / 2}\left\|U^{(N)}\right\|^{3}\right) \\
& \leqslant c_{58} d^{5 / 2} \tau^{2}\left(2^{-N / 2}+\frac{8 a_{5}^{3} \cdot 2^{3 u \mu-w N}}{d^{5} \tau^{3}}\right) \leqslant \frac{9 a_{5}^{3} c_{58} \cdot 2^{3 u \mu-w N}}{d^{2} \tau} \\
& \leqslant \frac{a_{5} \cdot 2^{3 u \mu-w N}}{2 c_{17} d^{2} \tau} \leqslant \frac{a_{5} \cdot 2^{3 u \mu-w N}}{2 d^{2} \tau} .
\end{aligned}
$$

From (10.1), (10.59), (10.61), (10.62) it follows that

$$
\left\|V^{(N)}\right\| \leqslant\left\|U^{(N)}\right\|+\left\|V^{(N)}-U^{(N)}\right\| \leqslant \frac{4 a_{5} \cdot 2^{u \mu+v N}}{d^{3 / 2} \tau} .
$$

Similarly, if we suppose that

$$
\mu \leqslant n \leqslant N-1,
$$

then from (10.1)-(10.3), (10.23), (10.26), (10.64) one can easily derive that

$$
\left\|U^{(n+1)}\right\| \leqslant \frac{4 c_{16}^{-1} a_{5} \cdot 2^{u \mu+v(n+1)}}{d^{3 / 2} \tau} \leqslant \frac{c_{57} \cdot 2^{n+1}}{d^{3 / 2} \tau} .
$$

Using the induction with respect to $n$, we shall prove that, if $\mu<N$, then, for $n=N-1, N-2, \ldots, \mu$,

$$
\begin{aligned}
\left\|\widetilde{\mathscr{U}}^{(n+1)}-\widetilde{\mathscr{V}}^{(n+1)}\right\| & \leqslant \frac{2 a_{5} \cdot 2^{3 u \mu-w(n+1)}}{c_{17} d^{2} \tau}, \\
\left\|U^{(n)}-V^{(n)}\right\| & \leqslant \frac{2 a_{5} \cdot 2^{3 u \mu-w n}}{d^{2} \tau}, \\
\left\|V^{(n)}\right\| & \leqslant \frac{4 a_{5} \cdot 2^{u \mu+v n}}{d^{3 / 2} \tau} .
\end{aligned}
$$

For $n=N$ the relations (10.67), (10.68) are already proved (see (10.62) and (10.63)). 
Assume that the inequalities (10.67), (10.68) are valid for $n=s+$ $1, \ldots, N$ and the inequality (10.66) holds for $n=s+1, \ldots, N-1, \mu \leqslant s \leqslant$ $N-1$. It suffices to prove the relations (10.66)-(10.68) for $n=s$.

Using (10.1)-(10.3), (10.19), (10.26), (10.60), (10.64), (10.65), (10.67), (10.68) and Lemma $9.1 \mathrm{~b}$ ), we obtain that in this case

$$
\begin{aligned}
\left\|\widetilde{\mathscr{U}}^{(s+1)}-\widetilde{\mathscr{V}}^{(s+1)}\right\| \leqslant & \frac{c_{58} d^{5 / 2} \tau^{2}}{2^{(s+1) / 2}}\left(1+\frac{\left\|\mathbf{U}^{(s+1)}\right\|^{3}}{2^{3(s+1) / 2} d^{1 / 2}}\right)+\frac{\left\|U^{(s+1)}-V^{(s+1)}\right\|}{8 c_{17}} \\
\leqslant & c_{58} d^{5 / 2} \tau^{2}\left(2^{-(s+1) / 2}+\frac{64 a_{5}^{3} \cdot 2^{3 u \mu-w(s+1)}}{c_{16}^{3} d^{5} \tau^{3}}\right) \\
& +\frac{a_{5} \cdot 2^{3 u \mu-w(s+1)}}{4 c_{17} d^{2} \tau} \leqslant \frac{65 c_{58} a_{5}^{3} \cdot 2^{3 u \mu-w(s+1)}}{c_{16}^{3} d^{2} \tau} \\
& +\frac{a_{5} \cdot 2^{3 u \mu-w(s+1)}}{4 c_{17} d^{2} \tau} \leqslant \frac{2 a_{5} \cdot 2^{3 u \mu-w(s+1)}}{c_{17} d^{2} \tau}
\end{aligned}
$$

The fulfilment of the conditions of Lemma $9.1 \mathrm{~b})$ can be easily deduced from the relations (10.1), (10.19), (10.26), (10.64), (10.65), (10.68) (with $n=s+1$ ) and from the definitions of $a_{5}, a_{6}, c_{74}$ (see (10.3)). The bound (10.69) yields (10.66) for $n=s$.

Applying Lemma 6.1 (see (6.41), (6.43) and (6.56)) and using (10.1), (10.2), (10.61), (10.62), (10.66) (with $n=s, \ldots, N-1$ ), we get

$\left\|U^{(s)}-V^{(s)}\right\| \leqslant \frac{c_{17}\left\|U^{(N)}-V^{(N)}\right\|}{2^{N-s}}+\sum_{k=s+1}^{N} \frac{c_{17}\left\|\widetilde{\mathscr{U}}^{(k)}-\widetilde{\mathscr{V}}^{(k)}\right\|}{2^{k-s}} \leqslant \frac{2 a_{5} \cdot 2^{3 u \mu-w s}}{d^{2} \tau}$

and, consequently,

$\left\|V^{(s)}\right\| \leqslant\left\|U^{(s)}\right\|+\left\|U^{(s)}-V^{(s)}\right\| \leqslant \frac{2 a_{5} \cdot 2^{u \mu+v s}}{d^{3 / 2} \tau}+\frac{2 a_{5} \cdot 2^{3 u \mu-w s}}{d^{2} \tau} \leqslant \frac{4 a_{5} \cdot 2^{u \mu+v s}}{d^{3 / 2} \tau}$.

This completes the proof of (10.66)-(10.68) for $n=s$.

It is evident that from (10.26), (10.42), (10.63), (10.68) it follows that

$$
\beta_{j}(N) \leqslant \mu .
$$

It is clear that the relations (10.52), (10.58), (10.70) imply

$$
2^{\beta_{j}(N)}+2^{\mu} \leqslant 2 \cdot 2^{\mu} \leqslant 2 \cdot 2^{\varkappa} \leqslant 2^{\beta_{i}(N-p)}+2 \cdot 2^{\nu} .
$$

Using (10.2), (10.3), (10.5), (10.24) (with $s=\mu),(10.26),(10.36)$, (10.38), (10.62), (10.66), we see that

$$
\begin{aligned}
\psi_{j}(N) & \leqslant \frac{a_{5} \cdot 2^{3 u \mu-w N}}{2 d^{2} \tau}+\sum_{n=\mu+1}^{N} \frac{2 a_{5} \cdot 2^{3 u \mu-w n}}{d^{2} \tau}+\frac{2 a_{5} \cdot 2^{\mu}}{d^{3 / 2} \tau}+4 \lambda \cdot 2^{\mu} \\
& \leqslant\left(2\left(1-2^{-w}\right)^{-1} a_{5}+4 \lambda\right) \cdot 2^{\mu} \leqslant 5 \lambda \cdot 2^{\mu} .
\end{aligned}
$$


The relations (10.40), (10.47), (10.71), (10.72) imply

$$
\begin{aligned}
\delta_{j}(N)+\rho_{j}(N) & \leqslant \delta_{i}(N-p)+5 \lambda\left(2^{\mu}+2^{\beta_{j}(N)}\right) \\
& \leqslant \delta_{i}(N-p)+\rho_{i}(N-p)+10 \lambda \cdot 2^{\nu} .
\end{aligned}
$$

From (10.48), (10.73) it is easy to derive the inequality (10.50) in the case $\nu \leqslant N$.

Let now $\nu=N+1$. From (10.15), (10.17), (10.31) it follows that

$$
S^{(N)}=U^{(N)}=U_{0}^{(N)}+V_{*}^{(N-p)} .
$$

Using (10.16), (10.38), we see that

$$
\psi_{j}(N) \leqslant\left\|S^{(N)}\right\|+\left\|S^{\{N\}}\right\|+\|T\| .
$$

By virtue of (10.34) we have

$$
\left\|S^{\{N\}}\right\| \leqslant\left\|S_{0}^{\{N\}}\right\|+\left\|T_{*}^{\{N-p\}}\right\| .
$$

It is clear that the relations $(10.14),(10.36),(10.37),(10.49),(10.74)-(10.76)$ imply

$$
\psi_{j}(N) \leqslant U+12 \lambda \cdot 2^{N} .
$$

From (10.47) and from definition of $\beta_{j}(N)$ it follows that $\beta_{j}(N) \leqslant N+1$ and

$$
\rho_{j}(N) \leqslant 10 \lambda \cdot 2^{N} .
$$

The relations (10.40), (10.48), (10.77), (10.78) imply

$$
\delta_{j}(N)+\rho_{j}(N) \leqslant \delta_{i}(N-p)+U+22 \lambda \cdot 2^{N}=\delta_{i}(N-p)+\gamma_{j}(N) .
$$

Thus, the inequality (10.50) is proved in the case $\nu=N+1$.

By construction (see (10.29)-(10.34), (10.38), (10.43)-(10.48)), the random variable $\gamma_{j}(N)$ is a function of

$$
\bigcup_{k=1}^{2^{N-p}}\left\{\widetilde{X}_{r}=Z_{r}^{[N]}, r=(k-1) \cdot 2^{p}+2, \ldots, k \cdot 2^{p}\right\}
$$

and the random variables $\delta_{i}(N-p), \rho_{i}(N-p)$ are functions of

$$
\left\{Y_{*, k}=Z_{(k-1) \cdot 2^{p}+1}^{[N]}, k=1, \ldots, 2^{N-p}\right\} .
$$

Since the sets $(10.80),(10.81)$ are disjoint sets consisting of jointly independent vectors (see (10.30)), the random variables $\gamma_{j}(N)$ and $\delta_{i}(N-p)+$ $\rho_{i}(N-p)$ are independent. Therefore, the inequality (10.51) follows from (10.50) and the proof of Lemma 10.1 is completed.

In this section we shall denote by $\varepsilon$ the quantity

$$
\varepsilon=\frac{a_{5}^{2}}{512 \lambda C_{2}^{2} d^{4} \tau^{2}} .
$$


Lemma 10.2. There exists a positive quantity $a_{7}$ depending only on $v$ and such that the following inequality is valid:

$$
\mathbf{E} \exp \left(\varepsilon \gamma_{j}(N)\right) \leqslant \exp \left(a_{7} p\left(\lambda d^{3 / 2} \tau\right)^{1 / v}\right) .
$$

P r o o f. Denote by

$$
\zeta=\mathbf{I}\{\nu=N+1\}
$$

the indicator function of the event $\{\nu=N+1\}$ (see (10.45)). Using (10.48) and the Hölder inequality, we obtain

$$
\mathbf{E} \zeta \exp \left(\varepsilon \gamma_{j}(N)\right) \leqslant\left(\mathbf{E} \zeta^{2}\right)^{1 / 2} \exp \left(22 \varepsilon \lambda \cdot 2^{N}\right)\left(\mathbf{E} e^{2 \varepsilon U}\right)^{1 / 2} .
$$

From the definitions of $U_{0}^{(n)}, S_{0}^{\{n\}}$ it follows that $U_{0}^{(N)}, S_{0}^{\{N\}}$ are sums of not more than $2^{N}$ independent random vectors with distributions $\mathscr{L}\left(\widetilde{X}_{k}\right) \in$ $\mathscr{A}_{d}(\tau)$ and covariance operators cov $\widetilde{X}_{k}=\mathbb{B}_{k}$ satisfying the condition (6.2) (see (10.31), (10.34)). Therefore, the relations (6.2), (6.16), (10.3), (10.8), (10.45), (10.84) and Lemma 2.6 with $B^{2} \leqslant C_{2}^{2} \cdot 2^{N}$ imply

$$
\begin{aligned}
\mathbf{E} \zeta^{2} & \leqslant \mathbf{P}\left\{\left|U_{0}^{(N)}\right|>\frac{a_{5} \cdot 2^{N}}{d^{2} \tau}\right\}+\mathbf{P}\left\{\left|S_{0}^{\{N\}}\right|>\frac{a_{5} \cdot 2^{N}}{d^{2} \tau}\right\} \\
& \leqslant 4 d \exp \left(-\min \left\{\frac{a_{5}^{2} \cdot 2^{N}}{4 C_{2}^{2} d^{4} \tau^{2}}, \frac{a_{5} \cdot 2^{N}}{4 d^{2} \tau^{2}}\right\}\right) \\
& =4 d \exp \left(-\frac{a_{5}^{2} \cdot 2^{N}}{4 C_{2}^{2} d^{4} \tau^{2}}\right) .
\end{aligned}
$$

It is clear that (see (10.49))

$$
\mathbf{E} e^{2 \varepsilon U} \leqslant\left(\mathbf{E} \exp \left(4 \varepsilon\left\|U_{0}^{(N)}\right\|\right) \mathbf{E} \exp \left(4 \varepsilon\left\|S_{0}^{\{N\}}\right\|\right)\right)^{1 / 2} .
$$

By virtue of (10.3), (10.5), (10.82) we have

$$
4 d^{1 / 2} \varepsilon \tau<1
$$

Using (10.88) and applying Lemma 2.6 with $B^{2} \leqslant C_{2}^{2} \cdot 2^{N}, h=4 d^{1 / 2} \varepsilon$, we see that

$$
\begin{aligned}
& \mathbf{E} \exp \left(4 \varepsilon\left\|U_{0}^{(N)}\right\|\right) \leqslant 2 d \exp \left(16 C_{2}^{2} d \varepsilon^{2} \cdot 2^{N}\right), \\
& \mathbf{E} \exp \left(4 \varepsilon\left\|S_{0}^{\{N\}}\right\|\right) \leqslant 2 d \exp \left(16 C_{2}^{2} d \varepsilon^{2} \cdot 2^{N}\right) .
\end{aligned}
$$

From (10.1), (10.3), (10.5), (10.6), (10.8), (10.82), (10.85)-(10.87), (10.89) it follows that

$$
\begin{aligned}
\mathbf{E} \zeta \exp \left(\varepsilon \gamma_{j}(N)\right) & \leqslant c d \exp \left(-\frac{a_{5}^{2} \cdot 2^{N}}{8 C_{2}^{2} d^{4} \tau^{2}}+22 \varepsilon \lambda \cdot 2^{N}+8 C_{2}^{2} d \varepsilon^{2} \cdot 2^{N}\right) \\
& \leqslant c d \exp \left(-\frac{a_{5}^{2} \cdot 2^{N}}{16 C_{2}^{2} d^{4} \tau^{2}}\right) \leqslant c d \exp \left(-\frac{p \cdot 2^{p}}{16 C_{2}^{2} d}\right) \\
& \leqslant c d \exp \left(-\frac{d^{1 / 2} \tau \lambda}{a_{5} C_{2}^{2}}\right) \leqslant c .
\end{aligned}
$$


Denote now by $m_{0}$ the minimal integer such that

$$
\frac{\tau^{2} d^{3} p}{2^{m_{0}-p}}<a_{6}
$$

It is evident that (10.3), (10.7)-(10.9), (10.91) imply $p \leqslant m_{0}=M+1 \leqslant N$. Using the definition of the random variable $\nu$ (see (10.46)), we get

$\mathbf{P}\{\nu=m+1\} \leqslant \sum_{n=m}^{N}\left(\mathbf{P}\left\{\left\|U_{0}^{(n)}\right\| \geqslant \frac{a_{5} \cdot 2^{u m+v n}}{d^{3 / 2} \tau}\right\}+\mathbf{P}\left\{\left\|S_{0}^{\{n\}}\right\| \geqslant \frac{a_{5} \cdot 2^{n}}{d^{3 / 2} \tau}\right\}\right)$,

$m=m_{0}, \ldots, N-1$. From the definitions of vectors $U_{0}^{(n)}, S_{0}^{\{n\}}$ it follows that they are sums of not more than $2^{n}$ independent random vectors with distributions $\mathscr{L}\left(\widetilde{X}_{k}\right) \in \mathscr{A}_{d}(\tau)$ and covariance operators cov $\widetilde{X}_{k}=\mathbb{B}_{k}$ satisfying the condition (6.2) (see (10.31), (10.34)). Therefore, for $m \geqslant m_{0}$, the relations (6.2), (6.16), (10.1), (10.3), (10.91) and Lemma 2.6 with $B^{2} \leqslant C_{2}^{2} \cdot 2^{n}$ imply

$$
\begin{aligned}
& \mathbf{P}\left\{\left\|U_{0}^{(n)}\right\| \geqslant \frac{a_{5} \cdot 2^{u m+v n}}{d^{3 / 2} \tau}\right\} \\
& \leqslant 2 d \exp \left(-\min \left\{\frac{a_{5}^{2} \cdot 2^{2 u m+2 v n}}{4 C_{2}^{2} d^{4} \tau^{2} \cdot 2^{n}}, \frac{a_{5} \cdot 2^{u m+v n}}{4 d^{2} \tau^{2}}\right\}\right) \\
& \quad=2 d \exp \left(-\frac{a_{5}^{2} \cdot 2^{2 u m+(2 v-1) n}}{4 C_{2}^{2} d^{4} \tau^{2}}\right), \quad n=m, \ldots, N
\end{aligned}
$$

and, analogously,

$$
\mathbf{P}\left\{\left\|S_{0}^{\{n\}}\right\| \geqslant \frac{a_{5} \cdot 2^{n}}{d^{3 / 2} \tau}\right\} \leqslant 2 d \exp \left(-\frac{a_{5}^{2} \cdot 2^{n}}{4 C_{2}^{2} d^{4} \tau^{2}}\right),
$$

$n=m, \ldots, N$. Taking into account (10.48), (10.84), we see that

$$
\mathbf{E}(1-\zeta) \exp \left(\varepsilon \gamma_{j}(N)\right) \leqslant \exp \left(10 \lambda \varepsilon \cdot 2^{m_{0}}\right)+\sum_{m=m_{0}+1}^{N} e^{10 \lambda \varepsilon \cdot 2^{m}} \mathbf{P}\{\nu=m\} .
$$

From (10.1), (10.3), (10.5), (10.82), (10.91) and from the definition of $p$ (see (10.6)) it follows that

$$
10 \lambda \varepsilon \cdot 2^{m_{0}} \leqslant \frac{10 a_{5}^{2}}{512 C_{2}^{2} d^{4} \tau^{2}} \cdot \frac{d^{3} \tau^{2} p \cdot 2^{p+1}}{a_{6}} \leqslant a p \cdot 2^{p} \leqslant a p\left(\lambda d^{3 / 2} \tau\right)^{1 / v} .
$$

Using (10.1)-(10.3), (10.5), (10.6), (10.82), (10.91)-(10.94), we obtain

$$
\begin{aligned}
& \sum_{m=m_{0}+1}^{N} e^{10 \lambda \varepsilon \cdot 2^{m}} \mathbf{P}\{\nu=m\} \\
& \quad \leqslant \sum_{m=m_{0}+1}^{N} \sum_{n=m-1}^{N} 4 d \exp \left(\frac{10 a_{5}^{2} \cdot 2^{m}}{512 C_{2}^{2} d^{4} \tau^{2}}-\frac{a_{5}^{2} \cdot 2^{2 u m+(2 v-1) n}}{8 C_{2}^{2} d^{4} \tau^{2}}\right)
\end{aligned}
$$




$$
\begin{aligned}
& \leqslant 4 d \sum_{m=m_{0}+1}^{N} \sum_{n=m-1}^{N} \exp \left(-\frac{a_{5}^{2} \cdot 2^{2 u m+(2 v-1) n}}{16 C_{2}^{2} d^{4} \tau^{2}}\right) \\
& \leqslant 4 d \sum_{m=m_{0}+1}^{\infty} c \ln ^{*}\left(a_{5}^{-1} d \tau\right) \exp \left(-\frac{a_{5}^{2} \cdot 2^{m}}{32 C_{2}^{2} d^{4} \tau^{2}}\right) \\
& \leqslant c d\left(\ln ^{*}\left(a_{5}^{-1} d \tau\right)\right)^{2} \exp \left(-\frac{a_{5}^{2} \cdot 2^{m_{0}}}{16 C_{2}^{2} d^{4} \tau^{2}}\right) \\
& \leqslant c d\left(\ln ^{*}\left(a_{5}^{-1} d \tau\right)\right)^{2} \exp \left(-\frac{p \cdot 2^{p}}{16 C_{2}^{2} d}\right) \\
& \leqslant c d\left(\ln ^{*}\left(a_{5}^{-1} d \tau\right)\right)^{2} \exp \left(-\frac{d^{1 / 2} \tau \lambda}{C_{2}^{2} a_{5}}\right) \leqslant c .
\end{aligned}
$$

The inequality (10.83) follows from (10.5), (10.6), (10.90), (10.95)-(10.97).

Lemma 10.3. Assume now (instead of (10.8) and (10.9)) that $N=M$. Then there exists a positive quantity $a_{8}$ depending only on $v$ and such that the following inequality is valid $\left(j=1, \ldots, 2^{M}\right)$ :

$$
\mathbf{E} \exp \left(\varepsilon\left(\rho_{j}(M)+\delta_{j}(M)\right)\right) \leqslant \exp \left(a_{8} p\left(\lambda d^{3 / 2} \tau\right)^{1 / v}\right) .
$$

P r o o f. Similarly to $(10.78)$, one can verify that $\rho_{j}(M) \leqslant 10 \lambda \cdot 2^{M}$. Therefore, using (10.38), we obtain

$$
\mathbf{E} \exp \left(\varepsilon\left(\rho_{j}(M)+\delta_{j}(M)\right)\right) \leqslant \exp \left(10 \varepsilon \lambda \cdot 2^{M}\right) \mathbf{E} e^{\varepsilon W},
$$

where $W=\|\widetilde{S}\|+\|T\|$. From the definitions of $\widetilde{S}, T=T_{j}$ (see (5.7), (10.38)) it follows that they are sums of not more than $2^{M}$ independent random vectors $\widetilde{X}_{k}$ and $Y_{k}^{[M]}$ respectively. By analogy with (10.14), we have $\mathbf{P}\{\|T\| \leqslant$ $\left.4 \lambda \cdot 2^{M}\right\}=1$. Hence,

$$
\mathbf{E} e^{\varepsilon W} \leqslant \mathbf{E} e^{\varepsilon\|\widetilde{S}\|} \exp \left(4 \varepsilon \lambda \cdot 2^{M}\right) .
$$

Recall that $\operatorname{cov} \tilde{X}_{k}=\mathbb{B}_{k}, \mathscr{L}\left(\widetilde{X}_{k}\right) \in \mathscr{A}_{d}(\tau)$. Taking into account (10.88) and applying Lemma 2.6 with $B^{2} \leqslant C_{2}^{2} \cdot 2^{M}, h=d^{1 / 2} \varepsilon$, we see that

$$
\mathbf{E} e^{\varepsilon\|\widetilde{S}\|} \leqslant 2 d \exp \left(C_{2}^{2} d \varepsilon^{2} \cdot 2^{M}\right)
$$

From (10.1), (10.3), (10.5)-(10.7), (10.82), (10.99)-(10.101) it follows that

$$
\begin{aligned}
\mathbf{E} \exp \left(\varepsilon\left(\rho_{j}(M)+\delta_{j}(M)\right)\right) & \leqslant 2 d \exp \left(14 \varepsilon \lambda \cdot 2^{M}+C_{2}^{2} d \varepsilon^{2} \cdot 2^{M}\right) \\
& \leqslant 2 d \exp \left(\frac{15 a_{5}^{2} \cdot 2^{M}}{512 C_{2}^{2} d^{4} \tau^{2}}\right) \leqslant 2 d \exp \left(a p \cdot 2^{p}\right) \\
& \leqslant 2 d \exp \left(a p\left(\lambda d^{3 / 2} \tau\right)^{1 / v}\right) .
\end{aligned}
$$

It is clear that (10.1), (10.5), (10.6), (10.102) imply (10.98). 
Write

$$
\begin{aligned}
b(N) & =\max _{1 \leqslant m \leqslant 2^{N}} \mathbf{E} \exp \left(\varepsilon\left(\delta_{m}(N)+\rho_{m}(N)\right)\right), \\
b(N-p) & =\max _{1 \leqslant m \leqslant 2^{N-p}} \mathbf{E} \exp \left(\varepsilon\left(\delta_{m}(N-p)+\rho_{m}(N-p)\right)\right), \\
r(N) & =\max _{1 \leqslant m \leqslant 2^{N}} \mathbf{E} \exp \left(\varepsilon \gamma_{m}(N)\right) .
\end{aligned}
$$

One can verify that the definitions of $b(N), b(N-p)$ are good coordinated: if we begin with $2^{N_{0}}$ vectors, where $N_{0}=N-p$, then $b\left(N_{0}\right)=b(N-p)$ (see $(10.27),(10.38)$ ). Since $j, 1 \leqslant j \leqslant 2^{N}$, is arbitrary, we can easily derive from (10.51), (10.103) and Lemma 10.2 that

$$
b(N) \leqslant b(N-p) r(N) \leqslant b(N-p) \exp \left(a_{7} p\left(\lambda d^{3 / 2} \tau\right)^{1 / v}\right) .
$$

Continuing this procedure and taking into account (10.7)-(10.9), we obtain

$$
b(N) \leqslant b(M) \exp \left(a_{7}(N-M)\left(\lambda d^{3 / 2} \tau\right)^{1 / v}\right) .
$$

From (10.9), (10.98), (10.103), (10.105) it follows that

$$
b(N) \leqslant \exp \left(a_{9} N\left(\lambda d^{3 / 2} \tau\right)^{1 / v}\right)
$$

with $a_{9}=\max \left\{a_{7}, a_{8}\right\}$. Using (10.38), (10.103), (10.106), we obtain

$$
\begin{aligned}
& \mathbf{E} \exp (\varepsilon \Delta(\tilde{X}, \tilde{Y})) \leqslant \sum_{m=1}^{2^{N}} \mathbf{E} \exp \left(\varepsilon \delta_{m}(N)\right) \\
& \quad \leqslant \sum_{m=1}^{2^{N}} \mathbf{E} \exp \left(\varepsilon\left(\delta_{m}(N)+\rho_{m}(N)\right)\right) \leqslant 2^{N} \exp \left(a_{9} N\left(\lambda d^{3 / 2} \tau\right)^{1 / v}\right) \\
& \quad \leqslant \exp \left(a N\left(\lambda d^{3 / 2} \tau\right)^{1 / v}\right) .
\end{aligned}
$$

Assume now, instead of (10.8), that $N \leqslant M$ and let the vectors $\widetilde{X}_{k}$, $k=1, \ldots, 2^{M}$, be constructed with the help of the vectors $\tilde{Y}_{k}, k=1, \ldots, 2^{M}$, in the same way as the vectors $X_{k}^{*}=\tilde{X}_{(k-1) \cdot 2^{N-M}+1}, k=1, \ldots, 2^{M}$, was constructed earlier with the help of the vectors $Y_{k}^{*}=Z_{(k-1) \cdot 2^{N-M}+1}^{[M+p]}$, $k=1, \ldots, 2^{M}$. For $k=2^{N}+1, \ldots, 2^{M}$ we can take $\mathscr{L}\left(\widetilde{X}_{k}\right)=\mathscr{L}\left(\tilde{X}_{1}\right)$, $\mathscr{L}\left(\tilde{Y}_{k}\right)=\mathscr{L}\left(\tilde{Y}_{1}\right)$. Now, if we denote

$$
\Delta_{N}(\tilde{X}, \tilde{Y})=\max _{1 \leqslant j \leqslant 2^{N}}\left\|\sum_{k=1}^{j} \tilde{Y}_{k}-\sum_{k=1}^{j} \tilde{X}_{k}\right\|,
$$

then, using (10.38), (10.98), (10.103), (10.108), we obtain

$$
\begin{aligned}
\mathbf{E} \exp \left(\varepsilon \Delta_{N}(\tilde{X}, \tilde{Y})\right) & \leqslant \sum_{m=1}^{2^{N}} \mathbf{E} \exp \left(\varepsilon \delta_{m}(M)\right) \leqslant 2^{N} \exp \left(a_{8} p\left(\lambda d^{3 / 2} \tau\right)^{1 / v}\right) \\
& \leqslant \exp \left(a(N+p)\left(\lambda d^{3 / 2} \tau\right)^{1 / v}\right) .
\end{aligned}
$$


P r o of of $\mathrm{Th}$ e o r e $\mathrm{m}$ 1.3. Without loss of generality we suppose that $0<\alpha \leqslant \frac{1}{5}$ and $n=2^{N}$. Suppose that independent Gaussian vectors $Y_{1}, \ldots, Y_{2^{N}}$ are already constructed. According to Theorem 8.2, we can construct the independent random vectors $\tilde{Y}_{1}, \ldots, \tilde{Y}_{2^{N}}$ so that $\mathscr{L}\left(\tilde{Y}_{k}\right)=$ $\Psi\left(\mathscr{L}\left(\widetilde{\eta}_{k}\right)\right), k=1, \ldots, n$, and

$$
\mathbf{E} \exp \left(\frac{c_{53} \Delta(\tilde{Y}, Y)}{d^{5 / 2} \tau}\right) \leqslant \exp \left(c_{54}(N+1) \ln ^{*} d\right) .
$$

Then we construct the vectors $\tilde{X}_{1}, \ldots, \widetilde{X}_{2^{N}}$ as functions of $\tilde{Y}_{1}, \ldots, \widetilde{Y}_{2^{N}}$ as it was described above in this section.

Taking $v=2 /(3+\alpha)$, we see that $v$ satisfies (10.1) and

$$
v^{-1}=\frac{3+\alpha}{2} \text {. }
$$

The relations (10.1), (10.3), (10.111) show that $a, a_{5}, \ldots, a_{9}$ can be considered as quantities depending only on $\alpha, C_{1}$ and $C_{2}$.

Let $M$ be the maximal integer satisfying the inequality (10.7) with $a_{6}, p$ defined by (10.3), (10.6). Define $N$ as the minimal integer such that $n \leqslant 2^{N}$, if $n \leqslant 2^{M}$, and as the minimal integer satisfying the relations $n \leqslant 2^{N}$ and (10.8), if $n>2^{M}$. In both cases we have

$$
N \leqslant c \ln n+p .
$$

It is evident that from (10.1), (10.5), (10.6), (10.111) it follows that

$$
p \leqslant a_{10} \ln ^{*}(\lambda d \tau) \leqslant a_{11}\left(\lambda d^{3 / 2} \tau\right)^{\alpha / 2},
$$

where $a_{10}, a_{11}$ depend only on $\alpha$. The statement of Theorem 1.3 follows now from (10.5), (10.82), (10.107)-(10.113).

\section{Proof of Theorem 1.2.}

Theorem 11.1. Suppose that $\alpha>0, A>0$, and $\xi_{1}, \ldots, \xi_{s}$ are independent random vectors with $\mathbf{E} \xi_{j}=0, j=1, \ldots, s$. Assume that there exists a strictly increasing sequence of nonnegative integers $0=i_{0}<i_{1}<\cdots<$ $i_{n}=s$ satisfying the following conditions. Write

$$
\tilde{\xi}_{k}=A\left(\xi_{i_{k-1}+1}+\cdots+\xi_{i_{k}}\right), \quad k=1, \ldots, n,
$$

and suppose that the covariance operators $\operatorname{cov} \tilde{\xi}_{k}=\mathbb{B}_{k}$ satisfy Condition $\mathbf{B}_{2}$ and $\mathscr{L}\left(\tilde{\xi}_{k}\right) \in \mathscr{A}_{d}\left(C_{1}\right), k=1, \ldots, n$, where $C_{1}$ is involved in Condition $\mathbf{B}_{2}$. Then one can construct on a probability space a sequence of independent random vectors $X_{1}, \ldots, X_{s}$ and a sequence of independent Gaussian random vectors $Y_{1}, \ldots, Y_{s}$ so that $\mathscr{L}\left(X_{j}\right)=\mathscr{L}\left(\xi_{j}\right), \mathbf{E} Y_{j}=0, \operatorname{cov} Y_{j}=\operatorname{cov} X_{j}$, $j=1, \ldots, s$, and

$$
\mathbf{E} \exp \left(\frac{a_{12} A \Delta(X, Y)}{d^{9 / 2} \ln ^{*} d}\right) \leqslant \exp \left(a_{13} d^{3+\alpha} \ln ^{*} n\right)
$$


where $a_{12}, a_{13}$ are positive quantities depending only on $\alpha, C_{1}, C_{2}$. Moreover, if all moments of the third order of the vectors $\tilde{\xi}_{k}$ are equal to zero, then this assertion is valid without the factor $\ln ^{*} d$ in the denominator of the fraction in the left-hand side of (11.2).

For the proof of Theorem 11.1 we need the following Lemma 11.1 which is proved in [29, Lemma 14.1] or [31, Lemma 9.2], see as well the proof of Theorem 1.6 in [31].

Lemma 11.1. Let $\mathscr{L}(\xi) \in \mathscr{A}_{d}(1), \mathbf{E} \xi=0$ and $\operatorname{cov} \xi=\mathbb{I}_{d}$. Then there exist a random vector $\eta$ and absolute positive constants $c_{75}, c_{76} \geqslant 1$ such that

$$
\begin{gathered}
\mathbf{E} \eta=\mathbf{E} \xi=0, \quad \operatorname{cov} \eta=\operatorname{cov} \xi=\mathbb{I}_{d}, \\
\mathbf{P}\{|\eta| \leqslant \rho\}=1, \quad \rho=c_{75}(1+\ln d), \quad \mathscr{L}(\eta) \in \mathscr{A}_{d}\left(c_{76}\right),
\end{gathered}
$$

and, for all $u, v, w \in \mathbb{R}^{d}$,

$$
\mathbf{E}\langle\eta, u\rangle\langle\eta, v\rangle\langle\eta, w\rangle=\mathbf{E}\langle\xi, u\rangle\langle\xi, v\rangle\langle\xi, w\rangle .
$$

Moreover, if all moments of the third order of the vector $\xi$ are equal to zero, then the same assertion is valid with $\rho=c_{75}$.

$\mathrm{Pr}$ o of of $\mathrm{Th}$ e or e m 11.1. Consider the random vectors $\zeta_{k}=$ $\mathbb{B}_{k}^{-1 / 2} \tilde{\xi}_{k}$. Obviously, $\operatorname{cov} \zeta_{k}=\mathbb{I}_{d}$ and Condition $\mathbf{B}_{2}$ implies that $\left\|\mathbb{B}_{k}^{-1 / 2}\right\| \leqslant$ $C_{1}^{-1},\left\|\mathbb{B}_{k}^{1 / 2}\right\| \leqslant C_{2}$ (see (6.15), (6.18) and (6.21)). Therefore, by Lemma 2.4, we have $\mathscr{L}\left(\zeta_{k}\right) \in \mathscr{A}_{d}(1)$. Using Lemma 11.2 , we see that there exist random vectors $\eta_{k}$, which have the same moments of the first three orders as $\zeta_{k}$, $\mathscr{L}\left(\eta_{k}\right) \in \mathscr{A}_{d}\left(c_{76}\right), \mathbf{P}\left\{\left\|\eta_{k}\right\| \leqslant \rho \sqrt{d}\right\}=1$, where $\rho=c_{75}(1+\ln d)$. Put $\widetilde{\eta}_{k}=\mathbb{B}_{k}^{1 / 2} \eta_{k}$. The random vectors $\widetilde{\eta}_{k}$ have the same moments of the first three orders as $\tilde{\xi}_{k}, \mathbf{P}\left\{\left\|\widetilde{\eta}_{k}\right\| \leqslant C_{2} \rho \sqrt{d}\right\}=1$, and, by Lemma $2.4, \mathscr{L}\left(\widetilde{\eta}_{k}\right) \in$ $\mathscr{A}\left(c_{76} C_{2}\right)$. Thus, the random vectors $\tilde{\xi}_{1}, \ldots, \tilde{\xi}_{n}$ satisfy the conditions of Theorem 1.3 with

$$
\tau=\max \left\{1, C_{1}, c_{76} C_{2}\right\}, \quad \lambda=\max \left\{C_{2}, 1, C_{2} c_{75}(1+\ln d)\right\} \sqrt{d} .
$$

Therefore, for any $\beta>0$ one can construct on a probability space a sequence of independent random vectors $\widetilde{X}_{1}, \ldots, \widetilde{X}_{n}$ and a sequence of independent Gaussian random vectors $\tilde{Y}_{1}, \ldots, \tilde{Y}_{n}$ so that $\mathscr{L}\left(\tilde{X}_{k}\right)=\mathscr{L}\left(\tilde{\xi}_{k}\right)$, $\operatorname{cov} \widetilde{Y}_{k}=\operatorname{cov} \widetilde{X}_{k}, \mathbf{E} \tilde{Y}_{k}=0, k=1, \ldots, n$, and

$$
\mathbf{E} \exp (b \Delta(\tilde{X}, \tilde{Y})) \leqslant \exp \left(a_{15}\left(\lambda d^{3 / 2} \tau\right)^{3 / 2+\beta} \ln ^{*} n\right),
$$

where

$$
b=\frac{a_{14}}{\lambda d^{4} \tau^{2}}
$$

and $a_{14}, a_{15}$ are quantities depending only on $\beta, C_{1}, C_{2}$. Using the BerkesPhilipp [2, Lemma A1, p. 53] and (11.1), we can suppose without loss of generality that

$$
\begin{aligned}
\tilde{X}_{k} & =A\left(X_{i_{k-1}+1}+\cdots+X_{i_{k}}\right), \quad k=1, \ldots, n, \\
\tilde{Y}_{k} & =A\left(Y_{i_{k-1}+1}+\cdots+Y_{i_{k}}\right),
\end{aligned}
$$


where $\left\{X_{1}, \ldots, X_{s}\right\}$ and $\left\{Y_{1}, \ldots, Y_{s}\right\}$ are two collections of mutually independent random vectors with $\mathscr{L}\left(X_{j}\right)=\mathscr{L}\left(\xi_{j}\right), \mathscr{L}\left(Y_{j}\right) \in \mathfrak{G}_{d}, \operatorname{cov} Y_{j}=\operatorname{cov} X_{j}$, $\mathbf{E} Y_{j}=0$, for $j=1, \ldots, s$.

Write

$$
\begin{aligned}
& S_{j}=X_{1}+\cdots+X_{j}, \quad j=1, \ldots, s ; \quad S_{0}=T_{0}=0 \\
& T_{j}=Y_{1}+\cdots+Y_{j},
\end{aligned}
$$

and

$$
\begin{aligned}
& \Delta_{1}=\max _{0 \leqslant k<n} \max _{1 \leqslant m \leqslant i_{k+1}-i_{k}}\left\|X_{i_{k}+1}+\cdots+X_{i_{k}+m}\right\|, \\
& \Delta_{2}=\max _{0 \leqslant k<n} \max _{1 \leqslant m \leqslant i_{k+1}-i_{k}}\left\|Y_{i_{k}+1}+\cdots+Y_{i_{k}+m}\right\|, \\
& \Delta_{3}=\max _{1 \leqslant k \leqslant n}\left\|S_{i_{k}}-T_{i_{k}}\right\| .
\end{aligned}
$$

It is evident that from (1.1), (11.9)-(11.12) it follows that

$$
\Delta(X, Y) \leqslant \Delta_{1}+\Delta_{2}+\Delta_{3} .
$$

Moreover, the relations (1.1), (11.8), (11.9), (11.12) imply

$$
\Delta_{3}=A^{-1} \Delta(\tilde{X}, \tilde{Y}) \text {. }
$$

It is clear that we can take $a_{14}<\frac{1}{2}$. From (11.7) and from the conditions of Theorem 11.1 it follows that $0<d^{1 / 2} b \tau<\frac{1}{2}$. Recall that Gaussian distributions from $\mathfrak{G}_{d}$ belong to all classes $\mathscr{A}_{d}(\tau)$. Using (11.5), Condition $\mathbf{B}_{2}$ and Lemma 2.6 with $t=d^{1 / 2} b, B^{2} \leqslant C_{2}^{2}$, we have

$$
\mathbf{E} e^{b A \Delta_{l}} \leqslant n \cdot 3 d e, \quad l=1,2 .
$$

Now the statement of Theorem 11.1 follows easily from the relations (11.5)-(11.7) and (11.13)-(11.15) by an application of the Hölder inequality to the exponential moment $\mathbf{E} \exp (b A \Delta(X, Y) / 3)$.

The case when all moments of the third order of the vectors $\tilde{\xi}_{k}$ are equal to zero can be considered in a similar way (cf. Lemma 11.2).

$\mathrm{Pr}$ o of of $\mathrm{Th}$ e o r e m 1.2. Without loss of generality we assume that $\tau^{2}=2^{M} C_{1}^{2}$, and $l=2^{N}$, where $M$ and $N$ are positive integers and $M \leqslant N$. Consider the random vectors

$$
\tilde{\xi}_{k}=2^{-M / 2}\left(\zeta_{(k-1) \cdot 2^{M}+1}+\ldots+\zeta_{k \cdot 2^{M}}\right), \quad k=1, \ldots, n=2^{N-M} .
$$

These vectors satisfy the conditions of Theorem 11.1 with $A=D \cdot 2^{-M / 2}=$ $C_{1} D / \tau, n=2^{N-M}=C_{1}^{2} l / \tau^{2}$. It remains to apply this theorem.

Pro of of $\mathrm{Th}$ e or e $\mathrm{m}$ 1.1. It is clear that it suffices to consider the case $\tau=1$. Otherwise one should use the result for $\tau=1$ to the random variables $\xi_{j} / \tau, j=1, \ldots, s$ (obviously, $\mathscr{L}\left(\xi_{j} / \tau\right) \in \mathscr{S}_{1}(1)$ ). Thus, let $\tau=1$. Then, by Lemma $2.3, \mathscr{L}\left(\xi_{j}\right) \in \mathscr{B}_{1}(1)$. Hence, by Lemma $\left.2.2 \mathrm{a}\right)$, $\mathscr{L}\left(\xi_{j}\right) \in \mathscr{A}_{1}\left(c_{2}\right)$ and $\mathbf{E} \xi_{j}^{2} \leqslant 12$.

There exists an absolute constant $c_{77}$ such that the inequality (1.3) is valid for $B^{2} \leqslant c_{77}$ with some sufficiently small absolute constant $c_{1}$ if we 
construct the variables $X_{1}, \ldots, X_{s}$ and $Y_{1}, \ldots, Y_{s}$ arbitrarily, for example, to be jointly independent. Write $S=X_{1}+\cdots+X_{s}$. By the completeness of the classes $\mathscr{A}_{d}(\tau)$ with respect to convolution, we have $\mathscr{L}(S) \in \mathscr{A}_{1}\left(c_{2}\right)$. The rest of the proof in the considered case uses Lemma 2.6 and the Kolmogorov and Hölder inequalities. It is elementary, repeats the arguments of Sakhanenko [18, Section 3.4]) and therefore is omitted. In this case the inequality (1.3) only reflects the fact that the compared random sequences are both sufficiently small.

Let now $B^{2} \geqslant c_{77}$. Then one can show that there exists an absolute constant $c_{78}$ and a strictly increasing sequence of nonnegative integers $m_{0}=0$, $m_{1}, \ldots, m_{l}=s$ such that

$$
c_{78} \leqslant \mathbf{E} \zeta_{p}^{2} \leqslant 24, \quad \mathscr{L}\left(\zeta_{p}\right) \in \mathscr{A}_{1}\left(c_{2}\right),
$$

where

$$
\zeta_{p}=\xi_{m_{p-1}+1}+\xi_{m_{p-1}+2}+\cdots+\xi_{m_{p}}, \quad p=1, \ldots, l .
$$

It remains to use Theorem 1.4 with $d=D=1, C_{1}=c_{78}, C_{2}=24$ and $\tau=c_{2}$ taking into account that $B^{2} \geqslant c_{78} l$. One should also note that, using the Hölder inequality, we can transfer the large factors under exponential sign in the right-hand side of inequalities of type (1.8) to the denominator of the fraction in the left-hand side.

Acknowledgment. The main part of this research was done in Bielefeld University. I would like to thank Professor Friedrich Götze for his hospitality during my stay in Bielefeld and for useful discussions.

\section{REFERENCES}

1. Berger E. Fast sichere Approximation von Partialsummen unabhängiger und stationärer ergodischer Folgen von Zufallsvectoren. Dissertation. Göttingen: Universität Göttingen, 1982.

2. Berkes I., Philipp W. Approximation theorems for independent and weakly dependent random vectors. - Ann. Probab., 1979, v. 7, p. 29-54.

3. Боровков A.A. О скорости сходимости в принципе инвариантности. - Теория вероятн. и ее примен., 1973 , т. 18, в. 2, с. 217-234.

4. Csörgö M., Révész P. A new method to prove Strassen type laws of invariance principle. I; II. - Z. Wahrscheinlichkeitstheor. verw. Geb., 1975, B. 31, S. 255-259; 261-269.

5. Csörgö M., Révész P. Strong Approximations in Probability and Statistics. New York: Academic Press, 1981, 284 p.

6. Csörgö S., Hall P. The Komlós-Major-Tusnády approximations and their applications. - Austral. J. Statist., 1984, v. 26, № 2, p. 189-218.

7. Einmahl $U$. A useful estimate in the multidimensional invariance principle. - Probab. Theory Relat. Fields, 1987, v. 76, № 1, p. 81-101.

8. Einmahl $U$. Strong invariance principles for partial sums of independent random vectors. - Ann. Probab., 1987, v. 15, p. 1419-1440.

9. Einmahl $U$. Extensions of results of Komlós, Major and Tusnády to the multivariate case. - J. Multivariate Anal., 1989, v. 28, p. 20-68. 
10. Гихман И. И., Скороход А. В. Теория случайньгх процессов. Т. І. М.: Наука, 1971, $664 \mathrm{c}$.

11. Götze F., Zaitsev A. Yu. Multidimensional Hungarian construction for vectors with almost Gaussian smooth distributions. Preprint 97-071 SFB 343. Bielefeld: Universität Bielefeld, 1997, 29 p.

12. Komlós J., Major P., Tusnády G. An approximation of partial sums of independent RV's and the sample DF. I; II. - Z. Wahrscheinlichkeitstheor. verw. Geb., 1975, B. 32 , S. $111-131 ; 1976$, B. 34, S. 34-58.

13. Massart $P$. Strong approximation for multivariate empirical and related processes, via KMT construction. - Ann. Probab., 1989, v. 17, № 1, p. 266-291.

14. Philipp W. Almost sure invariance principles for sums of $B$-valued random variables. - Lecture Notes in Math., 1979, v. 709, p. 171-193.

15. Прохоров Ю. В. Сходимость случайньх процессов и предельные теоремы теории вероятностей. - Теория вероятн. и ее примен., 1956, т. 1, в. 2, с. 177-238.

16. Rosenblatt $M$. Remarks on a multivariate transformation. - Ann. Math. Statist., 1952, v. 23, p. 470-472.

17. Рудзкис P. Вероятности больших уклонений случайных векторов. - Литов. матем. сб., 1983, т. 23, № 1, с. 195-204.

18. Саханенко А.И. Скорость сходимости в принципе инвариантности для разнораспределенных величин с экспоненциальными моментами. - Труды СО АН CCCP, 1984, т. 3, с. 4-49.

19. Саулис Л. Большие уклонения для случайных векторов для некоторьг классов множеств. - Литов. матем. сб., 1983, т. 23, № 3, с. 142-154.

20. Sazonov V. V. Normal Approximation - Some Recent Advances. Berlin-Heidelberg: Springer-Verlag, 1981, 105 p.

21. Shao Qi-Man. Strong approximation theorems for independent random variables and their applications. - J. Multivariate Anal., 1995, v. 52, № 1, p. 107-130.

22. Скороход A.В. Исследования по теории случайньх процессов. Киев: Киевский гос. ун-т, 1961, $216 \mathrm{c}$.

23. Statulevičius V. A. On large deviations. - Z. Wahrscheinlichkeitstheor. verw. Geb., 1966, B. 6, H. 2, S. 133-144.

24. Yurinskii V.V. On the error of the Gaussian approximation to the probability of a ball. Unpublished manuscript.

25. Зайчев А.Ю. О гауссовской аппроксимации сверток при выполнении многомерных аналогов условий типа неравенства Бернштейна. Препринт Р-9-84. Ленинград: ЛОМИ, 1984, 54 с.

26. Зайцев А.Ю. Оценки расстояния Леви-Прохорова в многомерной центральной предельной теореме для случайных векторов с конечными экспоненциальными моментами. - Теория вероятн. и ее примен., 1986, т. 31, в. 2, с. 246-265.

27. Zaitsev $A$. Yu. On the Gaussian approximation of convolutions under multidimensional analogues of S. N. Bernstein inequality conditions. - Probab. Theory Relat. Fields, 1987, v. 74, № 4, p. 535-566.

28. Зайчев A.Ю. О связи между двумя классами вероятностных распределений. Кольца и модули. Предельные теоремы теории вероятностей. Т. 2. Ленинград: Ленинграпский ун-т, 1988, с. 153-158.

29. Zaitsev A. Yu. Multidimensional version of the results of Komlós, Major and Tusnády for vectors with finite exponential moments. Preprint 95-055 SFB 343. Bielefeld: Universität Bielefeld, 1995, $112 \mathrm{p}$.

30. Зайчев А.Ю. Оценки квантилей гладких условных распределений и многомерный принцип инвариантности. - Сиб. матем. журн., 1996, т. 37, № 4, с. 807-831.

31. Zaitsev A. Yu. Multidimensional version of the results of Komlós, Major and Tusnády for vectors with finite exponential moments. - ESAIM, Probab. Statist., 1998, v. 2, p. $41-108$. 